\title{
KAC-MOODY LIE ALGEBRAS AND SOLITON EQUATIONS
}

\author{
II. LAX EQUATIONS ASSOCIATED WITH $\boldsymbol{A}_{1}{ }^{(1)}$ \\ H. FLASCHKA*, A.C. NEWELL** and T. RATIU*** \\ Department of Mathematics and Program in Applied Mathematics, University of Arizona, Tucson, Arizona 85721, USA
}

Received 29 November 1982

Revised 20 June 1983

\begin{abstract}
The soliton equations associated with sl(2) eigenvalue problems polynomial in the eigenvalue parameter are given a unified treatment; they are shown to be generated by a single family of commuting Hamiltonians on a subalgebra of the loop algebra of sl(2). The conserved densities and fluxes of the usual ANKS hierarchy are identified with conserved densities and fluxes for the polynomial eigenvalue problems. The Hamiltonian structures of the soliton equations associated with the polynomial eigenvalue problems are given a unified treatment.
\end{abstract}

\section{Introduction}

\subsection{Background}

This paper is concerned with the role of Lie algebras in soliton theory. We concentrate on the soliton equations associated with the AKNS eigenvalue problem

$V_{x}(x, \zeta)=\left[\left(\begin{array}{cc}-\mathrm{i} & 0 \\ 0 & \mathrm{i}\end{array}\right) \zeta+\left(\begin{array}{cc}0 & q(x) \\ r(x) & 0\end{array}\right)\right] V(x, \zeta)$

and on their relationship with the "loop algebra" of formal series $\Sigma X_{k} \zeta^{-k}, X_{k} \in \operatorname{sl}(2, \mathbb{C})$. Our results, which are new even for this familiar example, will be described shortly. First, we will explain the motivation for our study.

There had been a long-standing belief that Lie algebras are important in soliton theory. Such results as could be obtained in the early days generally reflected little more than the fact that, for instance,

\footnotetext{
* Supported in part by DOA Contract DAAG29-82-K-0068 and NSF grant MCS-81-02748A01.

** Supported in part by DOA Contract DAAG29. 82-K-0068, NSF grant MCS75-07548A01, and ONR Contract N0014-76-C-0867.

*** Supported in part by DOA Contract DAAG2982-K-0068 and NSF grant MCS81-06142.
}

the matrices in (1) belong to sl(2) with $x$-dependent coefficients; the deeper theory of Lie algebras was not brought in. The situation has changed dramatically in the last two or three years, and the fundamental role of Lie algebras and their representations in soliton theory is now appreciated.

We find it useful to classify the current Liealgebraic explanations of soliton phenomena into two (admittedly oversimplified) types.

In "type I", the point of view is that soliton equations are evolution equations for functions of $x$. Lie algebras arise because one looks at matrixfunctions of $x$, and they play the role of phase space for the evolution equations. These explanations have proved to be very successful in classifying Lax equations $\dot{L}=[B, L]$ for scalar operators, and in unravelling the Hamiltonian structure (in a functional-derivative sense) of those equations. See $[1,2,3]$.

The "type II" explanations - at least when they introduce Lie algebras - do not single out the variable $x$, and indeed do not deal with evolution equations. The basic object is the $\tau$-function, alias Hirota's new dependent variable [4]. It is a function of infinitely many variables: the original $x$, and infinitely many time variables, one for each equation in the soliton hierarchy. The phase space, such as it is, consists of $\tau$-functions, and as far as is 
known, is not equipped with a Poisson bracket. All these variables play similar roles. The Lie algebra, rather than providing the phase space, now acts on it as an algebra of symmetries. In going from type I to type II, one moves from a Lie algebra to a representation space. This kind of explanation makes sense out of Hirota's method and Bäcklund transformations. See the original work in [5].

It seemed puzzling to us that there were two different manifestations of the same Lie algebra. The ideal theory of soliton equations, therefore, should have the capacity to meld these two points of view. Furthermore, it should also permit one to deduce all the miracles of soliton theory completely systematically from a single starting point. Amongst the more spectacular of these miracles we count:

1) nontriviality of the Wahlquist-Estabrook prolongation;

2) existence of infinitely many local conservation laws;

3) the auxiliary spectral problem;

4) existence of a natural Hamiltonian structure;

5) Bäcklund and Miura transformations;

6) Hirota bilinearization;

7) the Painlevé property.

Our overall project is to incorporate all these features into a systematic and coherent picture. We still have a way to go before realizing our goals, but we do have results we find interesting, and we believe that our point of view will turn out to be correct for a comprehensive soliton theory. In this paper, we will explain our point of view and describe new results that give validity to our approach independently of the wider framework into which we hope to place it eventually.

The precise results obtained in this paper are summarized next. After that, at the end of this introduction, we indulge in a little speculation and explain what we think might happen in the future.

It should be made clear once and for all that we

\footnotetext{
* The unfortunate use of $\left({ }^{-i} i\right)$ in place of the more sensible $\left({ }^{\prime}-1\right)$ is a decade-long habit with some of us. The continuous spectrum of (1), in inverse scattering analyses, will lie on the real axis with our convention.
}

are interested in the formal properties of soliton equations. That means: we deal with symbols subject to certain algebraic rules. For example, we will use the language of differential algebras. Those consist of symbols such as $q, r, q_{x}, r_{x}, q_{x x}, \ldots$ that may be added and multiplied, and multiplied by constants, together with an operator $\mathrm{d} / \mathrm{d} x$ that takes $q$ to $q_{x}, q_{x}$ to $q_{x x}$, etc. This concept legitimizes the formal manipulations that are so useful in soliton theory. Another structure we need is the Lie algebra of formal series $\Sigma X_{k} \zeta^{-k}, X_{k} \in \operatorname{sl}(2, \mathbb{C})$. Convergence is not required. Throughout, it may be convenient to use language that sounds analytical, but no analytical properties are implied, and none are true without severe supplemental hypotheses $[6,7]$.

\subsection{Results}

Hoping to establish a direct connection between the type I and type II explanations mentioned above, we decided to reformulate the theory of AKNS soliton equations, as follows. The unknowns, $q(x)$ and $r(x)$, are usually taken to be functions of $x$, prescribed arbitrarily at time zero. We think of them instead as determined by a system of ordinary differential equations, in $x$, in infinitely many unknowns (rather like an analytic function of $x$ is determined by all its derivatives at $x=0$ ). The time-dependence is also given by a system of ordinary differential equations, in $t$. The use of this technique in formal soliton theory is new, as are the results we obtain from it, but a similar idea forms the basis for the Deift-Trubowitz study of KdV [8]. For them, the $x$-dependence of the initial $q(x)$ is (rigorously) determined by a Neumann oscillator system. We take it to be given formally by a kind of stationary equation. This will be explained presently.

We begin with a short review of the standard approach to the AKNS system (1).

The theory starts with

$$
V_{x}=\left(\begin{array}{cc}
-\mathrm{i} & \\
& \mathrm{i}
\end{array}\right) \zeta+\left(\begin{array}{l}
q \\
r
\end{array}\right) V \underset{\text { def }}{=} P V .
$$

$\zeta$ is the eigenvalue*; $q, r$ are functions of $x$, the 
"potentials." Adjoin to (1) a time-evolution for $V$,

$V_{t}=Q^{(N)} V$

where $Q^{(N)}=Q_{0} \zeta^{N}+Q_{1} \zeta^{N-1}+\cdots+Q_{N}$ is polynomial in $\zeta$, with coefficients being trace zero (i.e., sl(2)) matrices of polynomials in $q, r$ and their $x$-derivatives.

$$
V_{x t}=V_{t x} \text { implies }
$$

$P_{1}-Q_{x}^{(N)}+\left[P, Q^{(N)}\right]=0$

By setting the coefficient of each power of $\zeta$ equal to zero, one gets from (3) $\left(P_{0}=\left({ }^{-\mathrm{i}}{ }_{\mathrm{i}}\right)\right.$, $\left.P_{1}=\left({ }^{q}\right)\right)$ :

$$
\begin{array}{ll}
\zeta^{N+1}: & {\left[P_{0}, Q_{0}\right]=0,} \\
\zeta^{N}: & {\left[P_{0}, Q_{1}\right]+\left[P_{1}, Q_{0}\right]=Q_{0_{x}},} \\
\zeta^{N-k}: & {\left[P_{0}, Q_{k+1}\right]+\left[P_{1}, Q_{k}\right]} \\
& =Q_{k_{x}}(k<N) \\
\zeta^{0}: & P_{1_{t}}-Q_{N_{x}}+\left[P_{1}, Q_{N}\right]=0
\end{array}
$$

From (4), one finds $Q_{0}, \ldots, Q_{N}$ recursively. The $\zeta^{0}$-equation is then a p.d.e., $q_{t}=$ stuff, $r_{t}=$ stuff, where "stuff" means a polynomial in $q, r$ and their $x$ derivatives. Those are the soliton p.d.e.'s solved by the inverse scattering theory for (1).

There are many solutions $Q_{j}$ of (4), differing from each other by integration constants. The standard normalization is defined as follows: assign weight 1 to $q, r$ and each differentiation with respect to $x$. Then require that $Q_{j}$ be homogeneous of weight $j$. The first few $Q_{j}$ are

$$
\begin{aligned}
Q_{0} & =\left(\begin{array}{cc}
-\mathrm{i} \\
\mathrm{i}
\end{array}\right)\left(=P_{0}\right), \quad Q_{1}=\left(\begin{array}{c}
q \\
r
\end{array}\right)\left(=P_{1}\right), \\
Q_{2} & =\left(\begin{array}{cc}
-(\mathrm{i} / 2) q r & (\mathrm{i} / 2) q_{x} \\
-(\mathrm{i} / 2) r_{x} & (\mathrm{i} / 2) q r
\end{array}\right), \\
Q_{3} & =\left(\begin{array}{cc}
-\frac{1}{4}\left(q r_{x}-r q_{x}\right) & -\frac{1}{4} q_{x x}+\frac{1}{2} q^{2} r \\
-\frac{1}{4} r_{x x}+\frac{1}{2} q r^{2} & \frac{1}{4}\left(q r_{x}-r q_{x}\right)
\end{array}\right) .
\end{aligned}
$$

For later reference, we list the p.d.e.'s $(4)_{0}$ corre- sponding to $N=0,1,2,3$ in (2) (it is best to use a different symbol for the time variable in each, since eventually we will want to treat these equations simultaneously):

$$
\begin{aligned}
& (N=0) \quad q_{t_{0}}=-2 \mathrm{i} q, \quad r_{t_{0}}=2 \mathrm{i} r \\
& \text { (scaling of } q, r \text { ); } \\
& (N=1) \quad q_{t_{1}}=q_{x}, \quad r_{t_{1}}=r_{x} \\
& \text { (translation } x \mapsto x+t_{1} \text { ); } \\
& (N=2) \quad q_{t_{2}}=\frac{\mathrm{i}}{2} q_{x x}-\mathrm{i} q^{2} r, \\
& r_{t_{2}}=-\frac{\mathrm{i}}{2} r_{x x}+\mathrm{i} q r^{2} \\
& \text { (nonlinear Schrödinger); } \\
& (N=3) \quad q_{t_{3}}=-\frac{1}{4} q_{x x x}+\frac{3}{2} q q_{x} r, \\
& r_{t_{3}}=\frac{1}{4} r_{x x x}+\frac{3}{2} q r r_{x} \quad(\text { modified } \mathrm{KdV}) \text {, }
\end{aligned}
$$

In the usual formal theory, one now proves the following theorems:

(1) All equations $P_{t_{N}}=Q_{x}^{(N)}+\left[Q^{(N)}, P\right]$ commute, i.e., $P_{t_{N} t_{M}}=P_{t_{M^{\prime} N}}$.

(2) All these equations are Hamiltonian, i.e.,

$q_{t_{N}}=\frac{\delta \mathscr{F}_{N}}{\delta r}, \quad r_{t_{N}}=-\frac{\delta \mathscr{F}_{N}}{\delta q}$,

where $\mathscr{F}_{N}$ is a certain functional of $q, r$, and $\delta / \delta$ denotes the functional (or "Fréchet", or "variational") derivative. E.g., for $N=2, \quad \mathscr{F}_{2}=$ $\mathrm{i} / 6 \int\left(r_{x x} q+q_{x x} r-q_{x} r_{x}-3 r^{2} q^{2}\right) \mathrm{d} x$.

(3) The $\mathscr{F}_{N}$ are integrals $\int F_{N}$ of certain polynomials in $q, r$ and $x$-derivatives, and there is a relation of the form

$\frac{\partial F_{N}}{\partial t_{j}}=\frac{\partial G_{N j}}{\partial x}$

called a conservation law. The $G_{N j}$ are again differential polynomials. These results are usually proved via formal asymptotic expansions for the 
eigenfunction $V$ of (1) and/or a symbolic calculus for the inverse of $d / d x$.

We will get those results, and the analogous statements for any eigenvalue problem polynomial in $\zeta$,

$V_{x}=\left(\sum_{j=0}^{k} \zeta^{k-j} P_{j}\right) V$

from a single starting point: the Adler-KostantSymes theorem in the loop algebra $\operatorname{sl}(2) \otimes$ $\mathbb{C}\left[\zeta, \zeta^{-1}\right]$.

To this end, we first eliminate the special role assigned in the usual theory to " $x$ ", which means that we no longer take the eigenvalue problem (1) as basic. The idea is this.

All the $Q_{j}$ that could ever be generated from (4) can be determined from the single equation

$Q_{x}=[P, Q]$

in which $Q$ is now an infinite series $\sum_{j=0}^{\infty} Q_{j} \zeta^{-j}$. The coefficient of $\zeta^{-k}$ in (6) is $\left(P_{0}=Q_{0}, P_{1}=Q_{1}\right)$

$Q_{k_{x}}=\left[Q_{0}, Q_{k+1}\right]+\left[Q_{1}, Q_{k}\right]$,

which agrees with (4); there is, however, no $\zeta^{0}$ or $P_{t}$ term to end the recursion.

Suppose one now calculates all the $Q_{j}$ as matrices of (weighted homogeneous) differential polynomials in $x$. Let $q, r$ satisfy the $t_{2}$ or $t_{3}$ equation above. What equation does the series $Q$ obey?

One can verify that

$Q_{t_{2}}=\left[Q_{0} \zeta^{2}+Q_{1} \zeta+Q_{2}, Q\right]$,

$Q_{t_{3}}=\left[Q_{0} \zeta^{3}+\cdots+Q_{3}, Q\right]$,

and generally,

$Q_{t_{N}}=\left[Q^{(N)}, Q\right]$

Now we can get rid of $x$. $Q$ is a (formal) series $Q=\sum_{j=0}^{\infty} Q_{j} \zeta^{-j}=\sum_{j=0}^{\infty}\left(\begin{array}{cc}h_{j} & e_{j} \\ f_{j}-h_{j}\end{array}\right) \zeta^{-j}$, and it satisfies the equations

$Q_{t_{k}}=\left[Q^{(k)}, Q\right], \quad Q^{(k)}=\sum_{j=0}^{k} Q_{j} \zeta^{k-j}$

The $e_{j}, f_{j}, h_{j}$ are considered to be independent variables, and not differential polynomials. $x$ is now the time variable $t_{1}$ in (9), no different from $t_{2}$ or $t_{10}$ in its role in the theory.

An analogy with another feature of soliton theory may make this step clearer for some readers. Set $P_{t}=0$ in eq. (3). The result, $Q_{x}^{(N)}=\left[P, Q^{(N)}\right]$, is an o.d.e. in $x$ for time-independent, or stationary, solutions of (3). $q(x)$ and $r(x)$ determined from it are known to be multi-soliton or " $N$-gap" quasiperiodic initial conditions for all the equations (4), Our eq. (9), for $k=1$, is the stationary equation corresponding to $N=\infty$ in (3). Formally (but not analytically) it defines $q(x), r(x)$ as functions of $x=t_{1}$; they are " $\infty$-gap potentials."

Our first result will be seen to prove commutativity for all isospectral deformations of (1) or (5) in a single stroke.

Main result 1. Eq. (9) are commuting Hamiltonian flows on the Lie algebra $\left\{\Sigma_{0}^{\infty} X_{j} \zeta^{-j}, X_{j} \in \operatorname{sl}(2, \mathbb{C})\right\}$, with respect to a natural Lie-Poisson bracket.

Our next theorem shows that soliton equations in this generalized sense still have conservation laws. Indeed, the result provides, again in a single stroke, the local conservation laws for all isospectral deformations of all the eigenvalue problems (5).

Main result 2. There exist polynomials $F_{k j}$ in the $e_{i}, f_{i}, h_{i}$, such that

$\frac{\partial F_{k j}}{\partial t_{l}}=\frac{\partial F_{l k}}{\partial t_{j}}$.

First consequence. The usual conservation laws are

$\frac{\partial}{\partial t_{k}}($ density $)=\frac{\partial}{\partial x}$ (flux) : 
$\partial / \partial x$ is $\partial / \partial t_{1}$, i.e., $j=1$ in (10). But (10) suggests that if we care to view all variables as functions of $t_{j}$ for $j \neq 1$, we can do so and still have conservation laws. We return to this shortly.

Second consequence. More than (10) is in fact true:

$\frac{\partial F_{k j}}{\partial t_{l}}$ is symmetric under permutations of $k j l$

This suggests that $F_{k j}=\partial^{2} / \partial t_{k} \partial t_{j}$ of something. "Something" turns out to be $\log \tau$ of [5]. This will be shown in paper IV.

Suppose we now want to put $x$ back into the setup. We would do this by focusing on the case $k=1$ in (9); this just gives the recursion (7) from which the familiar $Q_{j}$ are determined in terms of $q$, $r, q_{x}, r_{x}, \ldots$ We would use the symbols $q$ and $r$ for $e_{1}$ and $f_{1}$, respectively. The first equations contained in (9) are $e_{1, t_{1}}=-2 \mathrm{i} e_{2}, f_{1, t_{1}}=2 \mathrm{i} f_{2}$. With $t_{1}=x$, this gives $e_{2}=(\mathrm{i} / 2) q_{x}, f_{2}=-(\mathrm{i} / 2) r_{x}$. Continuing in this way, we would view each individual equation in (9) as defining the next $e_{j}, f_{j}, h_{j}$ in terms of the preceding ones and their $t_{1}$-or $x$-derivatives, and arrive eventually at the usual expression for the $Q_{j}$. (This is done in more detail later on). We could, however, equally well focus on $k=2$ :

$Q_{t_{2}}=\left[Q_{0} \zeta^{2}+Q_{1} \zeta+Q_{2}, Q\right]$

This, too, leads to a recursion (similar to, but more complicated than (7)) and defines - it turns out all $Q_{j}$ in terms of $e_{1}, e_{2}, f_{1}, f_{2}$ and their $t_{2}$-derivatives. Here $e_{1}, f_{1}$, resp. $e_{2}, f_{2}$, are entries in $Q_{1}, Q_{2}$ - see above, eq. (8).

In effect, we have taken $t_{2}$ to be our $x$; this amounts to positing the eigenvalue problem

$$
\begin{aligned}
V_{x}= & {\left[\left(\begin{array}{cc}
-\mathrm{i} \\
& \mathrm{i}
\end{array}\right) \zeta^{2}+\left(\begin{array}{c}
e_{1} \\
f_{1}
\end{array}\right) \zeta\right.} \\
& \left.+\left(\begin{array}{cc}
-(\mathrm{i} / 2) e_{1} f_{1} & e_{2} \\
f_{2} & (\mathrm{i} / 2) e_{1} f_{1}
\end{array}\right)\right] V,
\end{aligned}
$$

and looking for its isospectral flows. Of course, there is nothing sacred about $t_{2}$.

Main result 3. Any $t_{N}$ may be taken as the special $x$. This amounts to positing the eigenvalue problem (5),

$V_{x}=\left(\sum_{j=0}^{N} \zeta^{N-j} Q_{j}\right) V$

$e_{1}, \ldots, e_{N}, f_{1}, \ldots, f_{N}$ in the $Q_{j}$ are $2 N$ potentials depending on $x$ (alias $t_{N}$ ); the $h_{j}$ turn out to be determined. All the flows (9) are Hamiltonian; the Hamiltonian for $(9)_{k}$ is $(2 \mathrm{i}) /(k+1) \int F_{N, k+1} \mathrm{~d} t_{N}$, where $F_{N, k+1}$ is the generalized density/flux from (10), viewed as differential polynomial in $e_{1}, \ldots, f_{N}$, with respect to $x=t_{N}$.

Actually, the correct conjugate variables turn out to be not the $e_{i}, f_{i}(i=1 \ldots N)$ ], but certain differential polynomials of them.

This theorem is the most important and surprising one; it should perhaps be explained in words. Main result 1 gave the soliton equations as Hamiltonian o.d.e.'s in $x$ and the various $t$ 's, but it must be remembered that the dependent variables $e_{j}, f_{j}, h_{j}$ are complex numbers and not functions of $x$. (One could think of them as the values of $q, r$ and all derivatives at a particular $x=x_{0}$, if that helps to clarify the idea). This Hamiltonian structure has nothing to do with the usual one $\left(q_{1}=\delta \int F / \delta r\right)$, and the Hamiltonians themselves have nothing to do with the familiar ones from inverse scattering theory. To recover the standard theory, one must view $e_{j}, f_{j}, h_{j}$ as polynomials in $q, r, q_{x}, r_{x}, \ldots$ as explained above. Result 3 says that the Hamiltonians then are given by integrals over certain fluxes from result 2 , and that indeed the Hamiltonians for any isospectral deformation of any eigenvalue problem (5) are given by fluxes from that one basic family.

These, then, are the main steps. We view the AKNS equations as commuting systems of o.d.e.'s for scalar unknowns rather than as evolution equations for functions of $x$. In that setting, we get a very simple proof of the commutativity of those 
flows. We then recover the conservation laws, and in fact discover much more structure to them than was previously known. When we try to return from our setup to the usual one, we find that our abstraction contains not only the AKNS soliton equations, but those of all polynomial eigenvalue problems (5) as well.

\subsection{Further discussion.}

In the summary above we have confined ourselves to the actual results presented. It is important to us, however, to view those results as part of a yet unfinished picture, and so we want to state briefly what must still be done and what we hope to do.

First of all, this paper has been kept on a concrete and computational level, in part because all our formulas derive from, and can be compared with, the well-studied AKNS theory. As a result, the role of the Lie algebra $\operatorname{sl}(2, \mathbb{C}) \otimes \mathbb{C}\left[\zeta, \zeta^{-1}\right]$ appears at times to be less central than it really is. At present, we can get main results 1 and 2, but not the crowning 3 , for any semisimple algebra in place of $\operatorname{sl}(2, \mathbb{C})$. This will be commented upon in the appropriate place. We still need to find a complete and general treatment for all twisted loop algebras. The computational evidence indicates that such a treatment will involve the full affine algebra, rather than just the loop algebra as in this paper, and this poses some additional obstacles.

We should explain that $\operatorname{sl}(2, \mathbb{C}) \otimes \mathbb{C}\left(\zeta, \zeta^{-1}\right)$ is the most obvious concrete example to choose because it turns up in connection with so many of the canonical solvable equations of mathematical physics. When we say 'turn up' we mean a lot more than observing that the Lax pairs of these equations can be expressed as systems of equations with $2 \times 2$ trace free matrices. In paper I, we show how the proper applications of the ideas of Wahlquist-Estabrook leads naturally to the choice of the phase space $\mathrm{sl}(2, \mathbb{C}) \otimes \mathbb{C}\left[\zeta^{-1}\right]$.

Our real goal as we indicated in subsection 1.1, is to see the $\tau$-function, Hirota's method, the vertex-operator representation of Bäcklund trans- formation, and the Painleve property as consequences of the framework introduced here. Throughout our calculations, we find formulas that beg for Hirota bilinearization, or for the introduction of a (formal) function that is known (from other work) to be $\tau$. We will point out some of those suggestive facts in due time. As one referee of an earlier version of this paper put it, however, the $\tau$-function is for us still a computational fact of life rather than a Lie-algebraic necessity.

\subsection{Related papers}

We want to draw attention to several related works about which we have learned in the course of our study.

The paper of Dhooghe [9] develops Hamiltonian systems on loop algebras essentially as we do here. Connections with evolution equations for functions of $x$ are established in a different way. The relations between conservation laws and variational Hamiltonians are given more emphasis in our work.

Segal and Wilson [6] put the $\tau$-function into a rigorous analytical framework. That paper, and a forthcoming work by Wilson (private communication) should go a long way towards uniting the new ideas of [5] with earlier soliton theory. We hope that our approach may eventually complement theirs by showing that all the main soliton ideas are related on a purely algebraic level as well as analytically.

A somewhat different analytical theory of $\tau$-functions is being worked out by $\mathbf{J}$. Palmer and D. Pickerell [7].

\section{Notation and basic algebraic ideas}

We will be dealing with two setups.

i) Lie algebras of formal power series, and vector fields on them. The concern is with-for instance-commutators of such vector fields, which make sense on a formal level, and not with their integral curves, which only exist under many additional hypotheses. 
ii) Differential algebras. Those are polynomial algebras generated by symbols $q, r, q_{x}, r_{x}, q_{x x}, \ldots$. They are equipped with a distinguished derivation $\partial$, thought of as $\mathrm{d} / \mathrm{d} x$ :

$\partial q=q_{x}, \quad$ etc.

We now set down our notation, and certain basic facts, for the two algebraic systems just mentioned.

\subsection{The loop algebra of $A_{1}^{(1)}$}

In this paper, the Lie algebra $\mathscr{G}$ of formal series $X=\sum_{j=-\infty}^{N} X_{j} \zeta^{j}, \quad X_{j} \in \mathrm{sl}(2, \mathbb{C}), \quad N<+\infty$,

will play the basic role. For a more general treatment, it becomes necessary to add to $\mathscr{G}$ two special elements (center and derivation), but in this paper they will not be required.

The commutator in $\mathscr{G}$ is defined by

$[X, Y]=\sum_{k} \sum_{i+j=k}\left[X_{i}, Y_{j}\right] \zeta^{k}$

it makes sense because the series $X, Y$ contain only finitely many positive powers of $\zeta$.

On $\mathscr{G}$, define the symmetric bilinear forms

$$
\langle X, Y\rangle_{k}=\sum_{i+j=k} \operatorname{Tr} X_{i} Y_{j}
$$

The case $k=0$ will be most important in this paper; $k=-1$ will play a role in paper III.

Certain subalgebras of $\mathscr{G}$ are needed:

$$
\begin{aligned}
& \mathscr{K}=\left\{\sum_{-\infty}^{-1} X_{j} \zeta^{j}\right\}, \\
& \mathscr{N}=\left\{\sum_{0}^{\infty} X_{j} \zeta^{j}\right\} \quad \begin{array}{c}
\text { (remember: only finitely many } \\
\text { positive powers of } \zeta)
\end{array}
\end{aligned}
$$

So $\mathscr{G}=\mathscr{K} \oplus \mathscr{N}$.

Let $\Pi_{\mathscr{K}}, \Pi_{\mathscr{K}}$ be the projections from $\mathscr{G}$ to $\mathscr{K}$ and
$\mathscr{N}$. The annihilators of $\mathscr{K}$ and $\mathscr{N}$ with respect to $\langle,\rangle_{0}$ are

$\mathscr{K}^{\perp_{0}}=\left\{\sum_{-\infty}^{0} X_{j} \zeta^{j}\right\}, \quad \mathscr{N}^{\perp_{0}}=\left\{\sum_{1}^{\infty} X_{j} \zeta^{j}\right\}$,

and with respect to $\langle,\rangle_{-1}$

$\mathscr{K}^{\perp_{-1}}=\mathscr{K}, \quad \mathscr{N}^{\perp_{-1}}=\mathscr{N}$.

We may and do identify the dual of $\mathscr{N}$ with $\mathscr{K}^{\perp}$. Depending on the choice of $\langle,\rangle_{k}, k=0$ or -1 , $\mathscr{N}^{*}$ can be realized concretely as $\mathscr{K}^{\perp_{0}}$ or $\mathscr{K}$. Likewise, $\mathscr{K}^{*}$ is identified either with $\mathscr{N}^{\perp_{0}}$ or $\mathscr{N}$. In this paper,

$\mathscr{N}^{*} \cong \mathscr{K}^{\perp_{0}}$

is used almost exclusively, and we henceforth omit the subscript 0 or -1 on $\langle$,$\rangle unless some$ confusion is possible.

Let $\phi$ be a $\mathbb{C}$-valued function on $\mathscr{G}$. Typically, $\phi(X)$ will be a polynomial in finitely many of the entries of the $X_{j}\left(X=\Sigma X_{j} \zeta^{j}\right)$. The tangent space to $\mathscr{G}$ at $X$ is identified, as usual, with $\mathscr{G}$ itself. Let $\dot{X} \in \mathscr{G} \cong T_{x} \mathscr{G}$, and take the derivative of $\phi$ in the direction $\dot{X}$ :

$\left.\frac{\mathrm{d}}{\mathrm{d} \epsilon} \phi(X+\epsilon \dot{X})\right|_{\epsilon=0}$

is a linear function of $\dot{X}$. The gradient of $\phi$ at $X$ is the element $\operatorname{grad} \phi(X) \in \mathscr{G}$ such that

$\left.\frac{\mathrm{d}}{\mathrm{d} \epsilon} \phi(X+\epsilon \dot{X})\right|_{\mathrm{c}=0}=\langle\operatorname{grad} \phi(X), \dot{X}\rangle$.

This definition has an analytical flavor, but it is possible to rephrase everything quite algebraically in terms of the polynomial algebra generated by the entries of the $X_{j}$.

\subsection{Differential algebra}

Let $\mathscr{B}$ be the polynomial algebra (over $\mathbb{C}$ ) generated by symbols $q_{j}^{(\alpha)}, r_{j}^{(\alpha)}(j=1, \ldots, N, \alpha \geqslant 0)$. Write $q_{j}^{(0)}=q_{j}, r_{j}^{(0)}=r_{j}$. Suppose there exists a 
derivation $\partial$ such that

$\partial: q_{j}^{(\alpha)} \rightarrow q_{j}^{(\alpha+1)}, \quad r_{j}^{(\alpha)} \rightarrow r_{j}^{(\alpha+1)}$

and such that $\partial: \mathbb{C} \rightarrow\{0\}$.

One thinks of $q_{j}, r_{j}$ as functions of a variable $x$, of $q_{j}^{(\alpha)}$ as $\mathrm{d}^{\alpha} q_{j} / \mathrm{d} x^{\alpha}$, and of $\partial$ as $\mathrm{d} / \mathrm{d} x$.

For $F \in \mathscr{B}$, define

$\frac{\delta F}{\delta s}=\sum_{i=0}^{\infty}\left(-\frac{\mathrm{d}}{\mathrm{d} x}\right)^{l} \frac{\partial F}{\partial s^{(n)}}$

$s$ is one of the $q_{j}, r_{j} . \delta F / \delta s$ is the functional (or variational) derivative of $F$ with respect to $s$. It can be proved [18] that $\delta F / \delta q_{j}=\delta F / \delta r_{j}=0$ for all $j$ if, and only if, $F \in \partial \mathscr{B}+\mathbb{C}$.

We now describe what we will call the variational Hamiltonian formalism.

Let $\overline{\mathscr{B}}$ be the quotient $\mathscr{B} /$ Image $\partial$. An equivalence class $F+$ Image $\partial$ will be denoted by $\bar{F} . \delta / \delta s$ $\left(s=q_{j}, r_{j}\right)$ acts from $\overline{\mathscr{B}}$ to $\mathscr{B}$,

$\frac{\delta}{\delta s}: \bar{F} \rightarrow \frac{\delta F}{\delta s}$.

This is well defined, since if $F_{1}, \quad F_{2} \in \bar{F}$, $\delta\left(F_{1}-F_{2}\right) / \delta s=0$ because $F_{1}-F_{2} \in$ Image $\partial$.

Let $\bar{F} \in \overline{\mathscr{B}}$. A derivation $\partial_{\bar{F}}$ is defined on $\overline{\mathscr{B}}$ as follows. Set

$\partial_{\bar{F}} q_{j}=\frac{\delta \bar{F}}{\delta r_{j}}, \quad \partial_{\bar{F}} r_{j}=-\frac{\delta \bar{F}}{\delta q_{j}}, \quad \partial_{\bar{F}}: \mathbb{C} \rightarrow\{0\}$

Extend $\partial_{\bar{F}}$ to all of $\mathscr{B}$ by requiring it to commute with $\partial: \partial_{\bar{F}} q_{j}^{(\alpha+1)}=\partial \partial_{\bar{F}} q_{j}^{(\alpha)}$, etc. Then $\partial_{\bar{F}}$ restricts to the quotient $\bar{\phi}$. The result, still denoted by $\partial_{\bar{F}}$ since only pedants will be confused, is the Hamiltonian derivation with Hamiltonian $\bar{F}$.

It is usually simpler to think of (11) as a system of partial differential equations, with $\partial_{\bar{F}} q_{j}$ being $\partial q_{j} / \partial t$ (but in some of the work described later on we found it helpful to be clear about what lives in which space). All the formulas, of course, are set up so as to mimick the usual derivatives of functionals of $q_{j}, r_{j} . F$ above, is concretely, the inte- grand of such a functional, and (11) is often written

$\frac{\partial q_{j}}{\partial t}=\frac{\delta}{\delta r_{j}} \int F \mathrm{~d} x, \quad \frac{\partial r_{j}}{\partial t}=-\frac{\delta}{\delta q_{j}} \int F \mathrm{~d} x$

Note that if $F$ is a perfect derivative, $F=G_{x}$, then $\int F \mathrm{~d} x=0$. Passage to $\bar{B}$ takes care of this.

It turns out, as expected, that the "Hamiltonian" of (11) is "constant": $\partial_{\bar{F}} \bar{F}=0$. A conserved density for (11) is a $\bar{G} \in \overline{\mathscr{B}}$ for which $\partial_{\bar{F}} \bar{G}=0$. There are alternative ways of saying this:

i) if $G \in \bar{G}$, then in $\mathscr{B}, \partial G / \partial t=\partial H / \partial x$ for some $H$ (the flux); to different $G$ 's correspond different $H$ 's.

ii) $\bar{F}$ and $\bar{G}$ are in involution with respect to the Poisson bracket in $\overline{\mathscr{B}}$,

$$
\begin{aligned}
& \{\bar{F}, \bar{G}\}=\sum_{j=1}^{N} \frac{\delta \bar{F}}{\delta r_{j}} \frac{\delta \bar{G}}{\delta q_{j}}-\frac{\delta \bar{F}}{\delta q_{j}} \frac{\delta \bar{G}}{\delta r_{j}}, \\
& \text { i.e., }\{\bar{F}, \bar{G}\}=0 .
\end{aligned}
$$

\section{Lax equations on $\mathscr{K}^{\perp}$}

In this section, we give two Lie-algebraic interpretations of the AKNS soliton equations in the version (9). We will not repeat the explanation of the relation between our abstract setup and the familiar language; that should be clear after reference to the introduction.

\subsection{1st Lie-algebraic interpretation}

The Lie-theoretic setup in this subsection is directly inspired by $\mathbf{M}$. Adler and P. van Moerbeke's paper [10] (which in turn goes back to Adler [11]). The decompositions we use, and the involution theorem, come from the section on "spinning tops" in [10]. We observed some time ago that "spinning tops" were relevant to stationary soliton equations, and what we develop below is a formal infinite-dimensional generalization of the stationary equations. See the comments in subsection 1.2, and also paper III of this series. $\mathscr{K}^{\perp}$, as the dual of $\mathcal{N}$, has a natural Poisson 
bracket, the so-called Lie-Poisson bracket. It is defined by $\dagger$

$$
\begin{aligned}
\{\phi, \psi\}(X)= & -\left\langle X,\left[\Pi_{\mathcal{N}} \operatorname{grad} \phi(X),\right.\right. \\
& \left.\left.\Pi_{\mathcal{N}} \operatorname{grad} \psi(X)\right]\right\rangle, \quad X \in \mathscr{K}^{\perp} .
\end{aligned}
$$

$\operatorname{grad} \phi(X)$ and grad $\psi(X)$ are computed with reference to the full Lie algebra $\mathscr{G}$, even though $\phi$ and $\psi$ may only be defined on $\mathscr{K}^{\perp}$.

To a function $\phi$ there is associated a Hamiltonian vectorfield

$$
\mathscr{X}_{\phi}(X)=-\Pi_{\mathscr{X} \perp}\left[\Pi_{\mathscr{N}} \operatorname{grad} \phi(X), X\right]
$$

Although the term "field" implies some smoothness, we only mean that $\mathscr{X}_{\phi}(X) \in T_{\chi} \mathscr{K}^{\perp} \cong \mathscr{K}^{\perp}$.

If $\phi$ is ad-invariant, meaning that

$[\operatorname{grad} \phi(X), X]=0, \quad X \in \mathscr{G}$,

then (13) becomes simpler:

$\mathscr{X}_{\phi}(X)=\left[\Pi_{\mathscr{X}} \operatorname{grad} \phi(X), X\right], \quad X \in \mathscr{K}^{\perp}$.

The Adler-Kostant-Symes theorem [11, 12, 13] says that if $\phi, \psi$ are both ad-invariant, (14), then

i) $\{\phi, \psi\} \equiv 0$ on $\mathscr{K}^{\perp}$;

ii) the vectorfields (15) commute.

(For proofs and further references, see the survey [14]).

The AKNS equations, in the form (9), arise via this theory from the simplest ad-invariant functions.

Let $\phi_{k}(X)=-\frac{1}{2}\left\langle S^{k} X, X\right\rangle$, where $S^{k}$ is the shift $\Sigma X_{j} \zeta^{j} \mapsto \Sigma X_{j} \zeta^{j+k}$, i.e., "multiplication by $\zeta^{k}$ ".

An easy calculation shows that

$\operatorname{grad} \phi_{k}(X)=-S^{k} X, \quad X \in \mathscr{G}$.

Since $\left[S^{k} X, X\right]=0, \phi_{k}(X)$ is ad-invariant. The formulas listed at the beginning immediately imply the following theorem:

\footnotetext{
$\uparrow$ Throughout subsection $3.1,\langle$,$\rangle means \langle,\rangle_{0}$ and $\perp$ means $\perp_{0}$.
}

Theorem 1. The functions $\phi_{k}(X)=$ $-\frac{1}{2}\left\langle S^{k} X, X\right\rangle, k \geqslant 0$, restricted to $X \in \mathscr{K}^{\perp}$, are in involution. Their Hamiltonian vector fields have the form

$\mathscr{X}_{\phi_{k}}(X)=-\left[\Pi_{\mathscr{X}} S^{k} X, X\right]$,

or, since $\left[S^{k} X, X\right]=0$,

$\mathscr{X}_{\phi_{k}}(X)=\left[\Pi_{. N} S^{k} X, X\right]$.

Note that (17) is essentially the version (9) of the AKNS equations, with $X$ standing for $Q$. We take $k \geqslant 0$ only, because otherwise $\left[\Pi_{\mathscr{X}} S^{k} X, X\right]=0$ for $X \in \mathscr{K}^{\perp}$, which is not interesting.

Warning: This theorem does not give the commutativity of the usual AKNS flows without a lot of further work. The Hamiltonians $\phi_{k}$ are completely different from the ones encountered in inverse scattering. See below, remark 2 following proposition 5 .

It will be useful to have a variety of formulas that allow one to find Poisson brackets between general, but explicitly given, functions. The explicit nature of the formulas that follow is a fortunate accident due to our choice of $\mathrm{sl}(2)$ as the basic Lie algebra. Even for $\operatorname{sl}(n)$ things get much more involved, and indeed the detailed information one can extract in the present case is a useful guide to the general situation. Remarks on a "coordinatefree" description are given at the end of this section.

We write $X \in \mathscr{K}^{\perp}$ as

$\sum_{j=0}^{\infty}\left(\begin{array}{cc}h_{j} & e_{j} \\ f_{j} & -h_{j}\end{array}\right) \zeta^{-j}$

and will work with the "coordinates on $\mathscr{K}^{\perp "}$, $e_{j}, f_{j}, h_{j}$, as well as with the series

$e=e(\zeta)=\sum_{j=0}^{\infty} e_{j} \zeta^{-j}$

$f$ or $f(\zeta)$, and $h$ or $h(\zeta)$ (defined similarly). These formal series make certain calculations easier. 
Proposition 1. $\phi_{k}(X)$ is the coefficient of $\zeta^{-k}$ in the series $-\left(h^{2}+e f\right)$,

$\phi_{k}(X)=-\sum_{i=0}^{k} h_{i} h_{k-i}+e_{i} f_{k-i}$

Proof. an easy calculation.

Proposition 2. The Poisson brackets between the $e_{j}, f_{j}$, and $h_{j}$ (viewed as functions on $\mathscr{K}^{\perp}$ ) are

$\left\{h_{i}, e_{j}\right\}=e_{i+j}$,

$\left\{h_{i}, f_{j}\right\}=-f_{i+j}$,

$\left\{e_{i}, f_{j}\right\}=2 h_{i+j}$.

Proof. For the moment, we write $e_{j}(X)$ to indicate that $e_{j}$ is a function of $X$. Now

$\left.\frac{\mathrm{d}}{\mathrm{d} \epsilon} e_{j}(X+\epsilon \dot{X})\right|_{\epsilon=0}=e_{j}(\dot{X})$,

(this is the $(1,2)$ entry in the coefficient of $\zeta^{-j}$ in $\dot{X} \in \mathscr{G})$. Since

$e_{j}(\dot{X})=\left\langle\zeta^{j}\left(\begin{array}{ll}0 & 0 \\ 1 & 0\end{array}\right), \dot{X}\right\rangle$

$\operatorname{grad} e_{j}(X)=\left(\begin{array}{cc}0 & 0 \\ 1 & 0\end{array}\right)^{j}$. Likewise,

$\operatorname{grad} f_{j}(X)=\zeta^{j}\left(\begin{array}{ll}0 & 1 \\ 0 & 0\end{array}\right)$,

$\operatorname{grad} h_{j}(X)=\frac{1}{2} \zeta^{j}\left(\begin{array}{rr}1 & 0 \\ 0 & -1\end{array}\right)$.

So

$$
\begin{aligned}
\left\{h_{i}, e_{j}\right\}(X) & =-\left\langle X,\left[\frac{1}{2} \zeta^{i}\left(\begin{array}{rr}
1 & 0 \\
0 & -1
\end{array}\right), \zeta^{j}\left(\begin{array}{ll}
0 & 0 \\
1 & 0
\end{array}\right)\right]\right\rangle \\
& =\left\langle X, \zeta^{i+j}\left(\begin{array}{ll}
0 & 0 \\
1 & 0
\end{array}\right)\right\rangle \\
& =e_{i+j}(X),
\end{aligned}
$$

and similarly for the other brackets.
The formulas (19) are easiest to use when expressed through "generating functions."

Proposition 3. Write $e=e(\zeta), e^{\prime}=e(\eta)$, and so forth for $f, h$, where $\zeta, \eta$ are formal parameters. Then

$\left\{h, e^{\prime}\right\}=\frac{\zeta^{-1} e-\eta^{-1} e^{\prime}}{\zeta^{-1}-\eta^{-1}}$,

$\left\{h, f^{\prime}\right\}=-\frac{\zeta^{-1} f-\eta^{-1} f^{\prime}}{\zeta^{-1}-\eta^{-1}}$,

$\left\{e, f^{\prime}\right\}=2 \frac{\zeta^{-1} h-\eta^{-1} h^{\prime}}{\zeta^{-1}-\eta^{-1}}$.

Proof.

$$
\begin{aligned}
\left\{h, e^{\prime}\right\} & =\sum_{i, j=0}^{\infty}\left\{h_{i}, e_{j}\right\} \zeta^{-i} \eta^{-j} \\
& =\sum_{i, j=0}^{\infty} e_{i+j} \zeta^{-i} \eta^{-j}=\sum_{k=0}^{\infty} e_{k} \sum_{i+j=k} \zeta^{-i} \eta^{-j} \\
& =\sum_{k=0}^{\infty} e_{k} \frac{\zeta^{-(k+1)}-\eta^{-(k+1)}}{\zeta^{-1}-\eta^{-1}} \\
& =\frac{\zeta^{-1} \sum_{k=0}^{\infty} e_{k} \zeta^{-k}-\eta^{-1} \sum_{k=0}^{\infty} e_{k} \eta^{-k}}{\zeta^{-1}-\eta^{-1}} \\
& =\frac{\zeta^{-1} e-\eta^{-1} e^{\prime}}{\zeta^{-1}-\eta^{-1}}
\end{aligned}
$$

etc.

Now, using the fact that the derivative of a function $\psi$ along a Hamiltonian vectorfield $x_{\phi}$ is given by $\{\psi, \phi\}$, we obtain equations for the derivatives $\partial e_{j} / \partial t_{k}, \ldots$, of $e_{j}, \ldots$, along the vectorfields $\mathscr{X}_{\phi_{k}}$.

Proposition 4.

$$
\begin{aligned}
& \frac{\partial e_{j}}{\partial t_{k}}=2 \sum_{i=0}^{\min (j-1, k)} h_{i} e_{k+j-i}-e_{i} h_{k+j-i}, \\
& \frac{\partial f_{j}}{\partial t_{k}}=2 \sum_{i=0}^{\min (j-1, k)} h_{i} f_{k+j-i}-e_{i} h_{k+j-i}, \\
& \frac{\partial h_{j}}{\partial t_{k}}=\sum_{i=0}^{\min (j-1, k)} e_{i} f_{k+j-i}-f_{i} e_{k+j-i} .
\end{aligned}
$$


Proof. Straightforward but tedious, using (19), or by directly calculating the $\zeta^{-j}$ component of $\left[\Pi_{,} S^{k} X, X\right]$.

Corollary. $h_{0}, e_{0}, f_{0}, h_{1}$ are independent of all $t_{k}$.

Proposition 5. Formulas (21) are expressed by generating functions, as follows:

$\left\{h^{2}+e f, e^{\prime}\right\}=\frac{2 \eta^{-1}}{\zeta^{-1}-\eta^{-1}}\left(e h^{\prime}-e^{\prime} h\right)$,

$\left\{h^{2}+e f, f^{\prime}\right\}=-\frac{2 \eta^{-1}}{\zeta^{-1}-\eta^{-1}}\left(f h^{\prime}-f^{\prime} h\right)$,

$\left\{h^{2}+e f, h^{\prime}\right\}=\frac{\eta^{-1}}{\zeta^{-1}-\eta^{-1}}\left(e^{\prime} f-e f^{\prime}\right)$.

Proof. Use Proposition 3. Note that the generating function for $\partial e_{j} / \partial t_{k}$ is $\left\{e^{\prime},-\left(h^{2}+e f\right)\right\}$, since the Hamiltonians $\phi_{k}$ are coefficients in $-\left(h^{2}+e f\right)$. Switching the order in \{\} removes the $(-)$ sign.

From (21) we easily get the following.

\section{Corollary.}

$\frac{\partial e_{j+1}}{\partial t_{k}}=\frac{\partial e_{k+1}}{\partial t_{j}}$,

$\frac{\partial f_{j+1}}{\partial t_{k}}=\frac{\partial f_{k+1}}{\partial t_{j}}$,

$\frac{\partial h_{j+1}}{\partial t_{k}}=\frac{\partial h_{k+1}}{\partial t_{j}}$.

Remark 1. This corollary is extremely important. It shows, for example, that $e_{j+1}, f_{j+1}, h_{j+1}$ are the $\partial / \partial t_{j}$ derivatives of functions of all the time variables. We will write (see eq. (29)) $h_{j+1}=$ $\partial / \partial t_{j} \cdot\left((\mathrm{i} / 2) \partial \ln \tau / \partial t_{1}\right) ; \quad \tau$ turns out to be the $\tau$-function of central importance to the theory. Similarly, the potentials $\sigma / \tau, \rho / \tau$ of which the sequences $e_{j}, f_{j}$ are gradients, are "auxiliary" $\tau$-functions and can be generated by applying Schlesinger transformations to the original $\tau$.
When one writes the equations of motion (21) in the potential variables $\tau, \sigma, \rho$ one obtains the Hirota equations. These results are elaborated on in paper IV.

Remark 2. The Hamiltonians $\phi_{k}$ are not the familiar Hamiltonians of inverse scattering theory, and carry little information. In inverse scattering, the Hamiltonians (integrals of conserved densities) have numerical values expressible in terms of the scattering data. One can, for example, relate the values of those Hamiltonians to soliton speeds. Our $\phi_{k}$, on the other hand, seem to carry no information in any numerical value that might be assigned to them. We now describe the implications of " $\phi_{k}=$ const."

Suppose we want to recover the usual AKNS $q$, $r, q_{x}, r_{x}, \ldots$ formulation of the soliton equations. We should then interpret the $t_{1}$-equation

$\frac{\partial X}{\partial t_{1}}=\left[\Pi_{,} S X, X\right]$

as the recursion relation it once was (see (4)). $X$ will be thought of as the $Q=\left({ }^{-i} i\right)+\left(r^{q}\right) \zeta^{-1}+\cdots$ of (6). So we should set $h_{0}=-i, h_{1}=e_{0}=f_{0}=0$ in $X$, and write $e_{1}=q, f_{1}=r$. Eq. (21) gives

$\frac{\partial e_{1}}{\partial t_{1}}=-2 \mathrm{i} e_{2} ;$

with $t_{1}=x$, this says $e_{2}=(\mathrm{i} / 2) q_{x}$. Likewise, $f_{2}=-(\mathrm{i} / 2) r_{x}$.

Next, we have $\partial e_{2} / \partial t_{1}=-2 \mathrm{i} e_{3}-2 e_{1} h_{2}$, or (i/2) $q_{x x}=-2 \mathrm{i} e_{3}-2 q h_{2}=0$. To find $e_{3}$ in terms of $q, r$, we need $h_{2}$.

$h_{2}$ comes from $\phi_{2}=$ const $=-c_{2}$, say:

$-2 \mathrm{i} h_{2}+h_{1}^{2}+e_{0} f_{2}+e_{1} f_{1}+e_{2} f_{0}=c_{2}$,

or

$h_{2}=c_{2}-\frac{\mathrm{i}}{2} q r$. 
Then $e_{3}=-\frac{1}{4} q_{x x}+\frac{1}{2} q^{2} r+\mathrm{i} c_{2} q$. We also get $f_{3}$ in an analogous way, find $h_{3}$ from $\phi_{3}=-c_{3}$, and so on.

Hence, a particular choice $\phi_{k}=-c_{k}(k \geqslant 2)$ only defines the $h_{k}$ as certain linear combinations of homogeneous differential polynomials in $q, r$. It has no influence whatsoever on the dynamics or on the character of the solutions $q(x, t), r(x, t)$; by a linear change of time variables, one can recover the homogeneous series $\dot{Q}$ familiar in the AKNS theory.

In order to set up a simple and direct correspondence between our phase-space and the differential algebra language, we will often find it convenient to restrict attention to a certain set $\mathscr{M} \subset \mathscr{K}^{\perp}$ :

\section{Definition.}

$$
\begin{aligned}
\mathscr{M} & =\left\{X \in \mathscr{K}^{\perp} \mid h_{0}=-\mathrm{i}, h_{1}=e_{0}=f_{0}=0, h^{2}+e f\right. \\
& =-1\} .
\end{aligned}
$$

Remark 3. Although they are never used in this paper, it is worth noting that we may recover the AKNS "eigenfunctions" $V$, and the equations they satisfy, as follows. The commuting family of flows (17),

$Q_{t_{k}}=\left[Q^{(k)}, Q\right]$

(we use the notation of (9)) admit a formal solution. Let

$Q=V Q_{0} V^{-1}$

it then follows that $Q_{0}$ is constant in all times $t_{k}$ if

$V_{t_{k}}=Q^{(k)} V-V N^{(k)}$,

and if $Q_{0}$ and $N^{(k)}$ (the latter is used to normalize the eigenfunctions in a suitable way) commute. In the present context, both $N^{(k)}$ and $Q_{0}$ are proportional to $H=\left(\begin{array}{cc}1 & 0 \\ 0 & -1\end{array}\right)$. Note from $Q=V Q_{0} V^{-1}$ that $h^{2}+e f=-\operatorname{det} Q=-\operatorname{det} Q_{0}=$ constant.

\subsection{2nd Lie algebraic interpretation}

There is another Hamiltonian structure that also gives the AKNS equations in the form (9). The Hamiltonians, however, are not those of subsection 3.1: the $\partial / \partial t_{k}$ vectorfield $\left[\Pi_{\mathcal{N}} S^{k} X, X\right]$ will come from the Hamiltonian $\phi_{k+1}$, not from $\phi_{k}$.

This second Hamiltonian structure is interesting for various reasons.

i) It arises from a quite different construction, namely, from a version of Kostant's translatedinvariant theorem $[12,14]$.

ii) Many soliton systems have two distinct Hamiltonian structures; there result the so-called Lenard relations that play an important role in the theory. Apparently, the AKNS system in its usual guise does not admit two (local) Hamiltonian structures. It is curious that our point of view provides two of them. We have looked for, but could not find, any more.

All arguments in this paper could be done with either structure; we will use interpretation 3.1, because the formulas are simpler.

The general Lie-algebra result is the following [14].

Let $\mathscr{G}=\mathscr{K} \oplus \mathscr{N}$, and let $\langle$,$\rangle be a non-$ degenerate symmetric, ad-invariant bilinear form on $\mathscr{G}$. There is a Hamiltonian structure on $\mathscr{K}^{\perp}$ (as in subsection 2.1); hence, by translation there will be one on the set $\mathscr{K}^{\perp}+\epsilon$, where $\epsilon \in \mathscr{G}$ is a fixed element. For certain $\epsilon$, special formulas hold.

Suppose

$\epsilon \perp[\mathscr{K}, \mathscr{K}]$ and $\epsilon \perp[\mathcal{N}, \mathscr{N}]$

Suppose furthermore that $\phi$ is ad-invariant on $\mathscr{G}$. The Hamiltonian vectorfield is then given by

$\mathscr{X}_{\phi}(X+\epsilon)=\left[\Pi_{\mathscr{X}} \operatorname{grad} \phi(X+\epsilon), X+\epsilon\right]$.

Moreover, if $\phi, \psi$ are ad-invariant then they are in involution with respect to this Poisson bracket on $\mathscr{K}^{\perp}+\epsilon$.

We apply this result with $\mathscr{G}, \mathscr{K}, \mathscr{N}$ as before, but with $\langle\rangle=,\langle,\rangle_{-1}$. For $\epsilon$, we take $\left(\begin{array}{cc}-\mathrm{i} & 0 \\ 0 & i\end{array}\right) \zeta^{0}$. 
It is easy to see that (24) holds for this $\epsilon$. As Hamiltonians, we take, as before, $\phi_{k}=$ $-\frac{1}{2}\left\langle S^{k} X, X\right\rangle_{0}=-\frac{1}{2}\left\langle S^{k-1} X, X\right\rangle_{-1}, k \geqslant 1$. The gradient of $\phi_{k}$ is now taken with respect to $\langle,\rangle_{-1}$, so $\operatorname{grad} \phi_{k}(X)=-S^{k-1} X$.

The Hamiltonian vectorfields (25) will then be, for $X \in \mathscr{K}$ :

$$
\begin{aligned}
\mathscr{X}_{\phi_{k}}(\epsilon+X) & =-\left[\Pi_{\mathscr{X}} S^{k-1}(\epsilon+X), \epsilon+X\right] \\
& =\left[\Pi_{\mathscr{N}} S^{k-1}(\epsilon+X), \epsilon+X\right] .
\end{aligned}
$$

There are two differences between this result and formula (17) in subsection 3.1:

1) Most importantly, the Hamiltonians are shifted: the vectorfield

$\left[\Pi_{.} S^{k} X, X\right], \quad X \in \mathscr{K}^{\perp_{0}} \quad$ or $\quad X \in \mathscr{K}^{\perp}+\epsilon$

has Hamiltonian $\phi_{k}$ in (17) and $\phi_{k+1}$ in (26).

2) In (17), the term $X_{0}$ in $X$ is constant by the corollary to prop. 4 . If we choose $X_{0}=\left({ }^{-i}{ }_{i}\right)$, the vectorfields (17) and (26) agree. The phase space $\mathscr{K}^{\perp_{-1}}+\epsilon$ is more restrictive in that the $\zeta^{0}$ coefficient is fixed once and for all, but that might actually be convenient for application to the AKNS system.

One can find analogs of the propositions in subsection 1. The basic formulas are

$\left\{h_{i}, e_{j}\right\}=e_{i+j-1}$,

$\left\{h_{i}, f_{j}\right\}=-f_{i+j-1}$,

$\left\{e_{i}, f_{j}\right\}=2 h_{i+j-1}$,

with the understanding that $h_{0}=-\mathrm{i}, e_{0}=0, f_{0}=0$. From these, the rest of the paper could be built up, but as stated above, we will use formulas (19) instead.

Remark. A coordinate-free description. The formulas of 3.1 can be adopted to loop algebras $g \otimes \mathbb{C}\left[\zeta, \zeta^{-1}\right], g$ a semisimple Lie algebra, in a fairly direct way. The basic Poisson brackets (19) could be thought of as dual to the commutation relations for a Weyl basis of $g$, but that seems to become very messy. Without choosing any particular basis of $g$, one can argue as follows.

Let $J_{\mu}, \mu=1, \ldots, R=$ rank of $g$, be a basis of homogeneous polynomials for the invariant polynomials on $g$. For $X=\Sigma X_{j} \zeta^{-j}, X_{j} \in g$, let $J_{\mu}^{k}(X)=(-1) \times \zeta^{-k}-$ coefficient of the formal series $J_{\mu}(X)$. The vectorfields

$\frac{\partial X}{\partial t_{\mu, k}}=\left[\Pi_{X} \operatorname{grad} J_{\mu}^{k}(X), X\right]$

then commute (analog of theorem 1).

Let $V \in g$. Then

$\frac{\partial}{\partial t_{\mu, k}}\left\langle\operatorname{grad} J_{\lambda}^{l+1}(X), V\right\rangle=\frac{\partial}{\partial t_{\lambda, l}}\left\langle\operatorname{grad} J_{\mu}^{k+1}(X), V\right\rangle$.

This generalizes the relations (23). Since $R=1$ for $g=\operatorname{sl}(2)$, the indices $\lambda, \mu$ may be dropped in this case, and $J^{k}$ is our earlier $-\phi_{k}$. With $V=\left({ }^{1}{ }_{-1}\right)$, $\left(\begin{array}{ll}0 & 1 \\ 0 & 0\end{array}\right),\left(\begin{array}{l}0 \\ 1\end{array}\right)$, we recover $(23)$.

Again, we will not spend much time on the general case, because we do not know a conceptual proof of theorem 2, section 7 .

\section{Conservation laws}

In this section, we derive conservation laws:

$\frac{\partial}{\partial t_{j}}($ density $)=\frac{\partial}{\partial x}($ flux $)$,

with $x=t_{1}$ as explained earlier. "Density" and "flux" are polynomials in the $e_{i}, f_{i}, h_{i}$. More generally, we obtain relations

$\frac{\partial}{\partial t_{j}}($ density $)=\frac{\partial}{\partial t_{l}}($ flux $)$

In our setup, none of the usual significance attaches to conservation laws. The variables are not functions of $x$, so $\int \ldots \mathrm{d} x$ has no meaning. It is all the more remarkable that conservation laws do exist, and in such generality. Moreover, the 
generalized conservation laws will be seen to be crucial to the variational Hamiltonian descriptions later on.

The formulas we want involve 3 indices. Correspondingly, there are generating series in 3 parameters: $\zeta, \eta, \xi$. We write $e=e(\zeta), e^{\prime}=e(\eta)$, $e^{\prime \prime}=e(\xi)$, and so on. Define

$$
\begin{aligned}
F(\eta, \xi)= & \frac{1}{2 h_{0}} \frac{\eta^{-1} \xi^{-1}}{\left(\eta^{-1}-\xi^{-1}\right)^{2}} \\
& \times\left[e^{\prime} f^{\prime \prime}+e^{\prime \prime} f^{\prime}+2 h^{\prime} h^{\prime \prime}-\left(h^{2}+e^{\prime} f^{\prime}\right)\right. \\
& \left.-\left(h^{\prime 2}+e^{\prime \prime} f^{\prime \prime}\right)\right]
\end{aligned}
$$

Lemma. $F(\eta, \xi)$ is a formal power series in $\eta^{-1}, \xi^{-1}$.

Proof. The only thing to check is that the expression inside [. . . is divisible by $\left(\eta^{-1}-\xi^{-1}\right)^{2}$. This is so because $[\ldots], \partial[\ldots] / \partial \eta$, and $\partial[\ldots] / \partial \xi$ all vanish when $\xi=\eta$.

Write $F(\eta, \xi)=\Sigma_{k, j=1}^{\infty} F_{k j} \eta^{-k} \xi^{-j}$. Since $F(\eta, \xi)=$ $F(\xi, \eta)$, we have this

Lemma.

$F_{k j}=F_{j k}$.

\section{Lemma.}

$\frac{\partial h_{j+1}}{\partial t_{k}}=\frac{\partial F_{k j}}{\partial t_{1}}$

Remark. Eqs. (29) are the usual AKNS conservation laws. The $h_{j+1}$ are the conserved densities, and the $F_{k j}$ are the corresponding fluxes.

Proof. From (22),

$\left\{h^{2}+e f, h^{\prime}\right\}=\frac{\eta^{-1}}{\zeta^{-1}-\eta^{-1}}\left(e^{\prime} f-e f^{\prime}\right)$.

On the other hand, $\partial \eta^{-1} F(\xi, \eta) / \partial t_{1}=$ $\left\{2 h_{0} h_{1}+e_{0} f_{1}+e_{1} f_{0}, \eta^{-1} F(\zeta, \eta)\right\}$ can be evaluated from formulas (19). One finds that

$$
\left\{h^{2}+e f, h^{\prime}\right\}=\frac{\partial}{\partial t_{1}} \eta^{-1} F(\zeta, \eta)
$$

or

$\sum \frac{\partial h_{j}}{\partial t_{k}} \zeta^{-k} \eta^{-j}=\frac{\partial}{\partial t_{1}} \sum F_{k j} \zeta^{-k} \eta^{-(j+1)}$,

from which the desired result is read off.

\section{Proposition 6.}

$$
\begin{aligned}
F_{k l}= & \frac{1}{2 h_{0}} \sum_{i=0}^{k-1}(k-i)\left(2 h_{i} h_{k+l-i}\right. \\
& \left.+e_{i} f_{k+l-i}+f_{i} e_{k+l-i}\right)+k \phi_{k+l} .
\end{aligned}
$$

Proof. The coefficient of $x^{k}$ in a formal series $(x-y)^{-2} \Sigma a_{i j} x^{i} y^{j}$ is

$\left.\frac{1}{k !} \frac{\partial^{k}}{\partial x^{k}}(x-y)^{-2} \sum a_{i j} x^{i} y^{j}\right|_{x=0}$,

which is calculated to be

$$
\begin{aligned}
& \sum_{\alpha=0}^{k}(k-\alpha+1) \sum_{j=0}^{\infty} a_{\alpha j} y^{j-(k-\alpha+2)} \\
& =\sum_{l=0}^{\infty}\left(\sum_{\alpha=0}^{k}(k-\alpha+1) a_{\alpha, l+k-\alpha+2}\right) y^{l}
\end{aligned}
$$

Now set $a_{i j}=\left(1 / 2 h_{0}\right)\left(2 h_{i-1} h_{j-1}+e_{i-1} f_{j-1}+\right.$ $\left.e_{j-1} f_{i-1}+\phi_{i-1} \delta_{0, j-1}+\phi_{j-1} \delta_{0, i-1}\right)$ to get the result $\left(a_{0 j}=a_{i 0}=0\right)$.

Remark. Since $\partial \phi_{k} / \partial t_{j}=0$ for any $j$, the $\phi_{k+l}$ term in (30) may be left off without affecting any formula with the exception of (28).

Remark. $F_{1, l}=h_{l+1}+\phi_{l+1}$. When combined with (29), this just says that $\partial h_{l+1} / \partial t_{1}=\partial h_{l+1} / \partial t_{1}$.

From (22), we get 


\section{Proposition 7.}

$$
\begin{aligned}
& \left\{h^{2}+e f, F(\eta, \xi)\right\} \\
& =\frac{\zeta^{-1} \eta^{-1} \xi^{-1}}{h_{0}\left(\xi^{-1}-\eta^{-1}\right)\left(\zeta^{-1}-\xi^{-1}\right)\left(\zeta^{-1}-\eta^{-1}\right)} \\
& \quad \times\left[h\left(e^{\prime} f^{\prime \prime}-e^{\prime \prime} f^{\prime}\right)+h^{\prime}\left(e^{\prime \prime} f-e f^{\prime \prime}\right)\right. \\
& \left.\quad+h^{\prime \prime}\left(e f^{\prime}-e^{\prime} f\right)\right] .
\end{aligned}
$$

The only point of this result is: the right side is symmetric in $\zeta, \eta, \xi$. Hence, so is the left side. Therefore, we have this

Corollary. $\partial F_{k j} / \partial t_{i}$ is symmetric under permutations of $i j k$.

Now there are the two consequences already mentioned in the introduction:

(1) By symmetry, $\partial F_{k j} / \partial t_{i}=\partial F_{i k} / \partial t_{j}$ etc. This generalizes the conservation law (29) to arbitrary $t_{j}$ instead of $t_{1}$.

(2) The symmetry of $\partial F_{k j}$ suggests the introduction of a function of the $t$ 's - we will call it $\log \tau\left(t_{1}, t_{2}, \ldots\right)$ - such that

$F_{k j}=\frac{\partial^{2} \log \tau}{\partial t_{k} \partial t_{j}}$

This direction will be explored in paper IV.

\section{Remark. A coordinate-free description.}

We continue the notation of the remark at the end of section 3. In the more general case $g \otimes \mathbb{C}\left[\zeta, \zeta^{-1}\right]$, set

$$
F_{\mu, k: R, l}(X)=\left\langle\left[D, \Pi_{\mathcal{N}} \operatorname{grad} J_{\mu}^{k}(X)\right], \operatorname{grad} J_{\lambda}^{l}(X)\right\rangle .
$$

Here $D$ is the derivation $\zeta \mathrm{d} / \mathrm{d} \zeta$. One can then verify:

(i) "Symmetry": $F_{\mu, k: \lambda, l}=F_{\lambda, l ; \mu, k}+$ a term annihilated by all $\partial / \partial t_{v, m}$.

(ii) Analog of (29):

$$
\frac{\partial}{\partial t_{v, 1}} F_{\mu, k ; ; ; i}(X)=\frac{\partial}{\partial t_{\mu, k}}\left\langle\operatorname{grad} J_{i}^{l+1}(X), \operatorname{grad} J_{\mu}^{k+1}\left(X_{0}\right)\right\rangle
$$

( $X_{0}$ is the coefficient of $\zeta^{0}$ in $X$; it is taken to lie in a fixed Cartan subalgebra of $g$ )

(iii) Analog of the last corollary:

$\frac{\partial}{\partial t_{v, m}} F_{\mu, k ; \lambda, l}=\frac{\partial}{\partial t_{\mu, k}} F_{v, m ; i, l}$.

Here, incidentally, is another reason for our avoiding general semisimple Lie algebras. The definition of $F_{\mu, k ; \beta, l}$ makes it embarrassingly clear that everything should take place on the affine algebra $g \otimes \mathbb{C}\left[\zeta, \zeta^{-1}\right]+$ center + derivation. Our attempts to put the Hamiltonian system directly on this extension of the loop algebra have failed so far. It should be remarked that all formulas involving fluxes of conservation laws or $\tau$-functions implicity bring in the derivation $\zeta \mathrm{d} / \mathrm{d} \zeta$. For example, our $F_{k j}$ 's above can be written (eq. (30))

$$
\begin{aligned}
F_{k j}= & \operatorname{Tr}\left(k Q_{0} Q_{k+j}+(k-1) Q_{1} Q_{k+j-1}+\cdots\right. \\
& \left.+Q_{k-1} Q_{j+1}\right)+k \phi_{k+l}
\end{aligned}
$$

The $k,(k-1), \ldots$ came from $\zeta \mathrm{d} \zeta^{k} / \mathrm{d} \zeta=k \zeta^{k}$, etc. Or, the "vertex operator" of [5] has the general form

$\exp \left(\sum \zeta^{k} t_{k}+\sum \frac{1}{k} \zeta^{-k} \frac{\partial}{\partial t_{k}}\right)$

Again, the $1 / k$ comes from $\zeta \mathrm{d} / \mathrm{d} \xi$, but in all studies to date, the derivation (and hence the complete affine Lie algebra) has only played an implicit role.

We consider this to be the main obstacle to a comprehensive theory.

\section{Vectorfields that commute with $\mathscr{X}_{\phi_{N}}$}

We now turn to the translation of the Liealgebraic framework above to the more familiar language of differential algebra. At the end of section 4.1, we saw that it was natural to introduce new variables (on the subset $\mathscr{M} \subset \mathscr{K}^{\perp}$, at least):

$e_{1}=q, \quad f_{1}=r, \quad e_{2}=\frac{\mathrm{i}}{2} q_{x}, \quad f_{2}=\frac{\mathrm{i}}{2} r_{x}, \ldots$ 
There is no problem as long as $q, q_{x}, q_{x x}$ etc., are thought of as mere symbols. If $q_{x}$ is to mean $\partial q / \partial x$, however, a difficulty does arise.

Let $\psi$ be any polynomial in the $e$ 's, $f$ 's and $h$ 's. If it be taken as Hamiltonian, there will be the equations

$\dot{e}_{j}=\left\{e_{j}, \psi\right\}$

and so on for $f, h$.

These will make sense in the variational framework, where $e_{1}=q$ and $f_{1}=r$ are functions of $x$, when relations like

$$
\begin{aligned}
& \left(\frac{\partial e_{1}}{\partial x}\right)=\frac{\partial \dot{e}_{1}}{\partial x}, \\
& \left(\frac{\partial \dot{e}_{1}}{\partial x}\right)=\frac{\partial \ddot{e}_{1}}{\partial x},
\end{aligned}
$$

hold. That is necessary because all Hamiltonian derivations

$\dot{q}=\frac{\delta F}{\delta r}, \quad \dot{r}=-\frac{\delta F}{\delta q}$

commute with $\partial / \partial x$. Hence, a vectorfield on $\mathscr{K}^{\perp}$ can be meaningful in the differential algebra interpretation of AKNS only when it commutes with $\partial / \partial x$, i.e., with the vectorfield $\mathscr{X}_{\phi_{1}}$.

We will want to have the freedom of choosing any $t_{N}$ as the distinguished $x$, so we must understand vectorfields on $\mathscr{K}^{\perp}$ commuting with a fixed $t_{N}$. That is the question taken up in this section. From here on, our results are known only for sl(2), and we can make no more comments about other Lie algebras.

First, we prove that the Hamiltonians $\phi_{k}$ are a maximal commuting family in a very strong sense.

Proposition 8. Let $\psi$ be a polynomial in $e$ 's, $f$ 's, and $h$ 's that commutes with a fixed $\mathscr{X}_{\phi_{N^{*}}}$. Then $\psi$ is a polynomial of the $\phi_{k}, k \geqslant 1$, and of $h_{0}, h_{0}^{-1}, e_{0}$, $f_{0}$.

Proof. Instead of $e_{j}, f_{j}, h_{j}, j \geqslant 0$, it will be con- venient to use, as coordinates on $\mathscr{K}^{\perp}$,

$e_{j}, f_{j}, j \geqslant 0 ; h_{0} ; \phi_{j}, j \geqslant 1$.

To see that this is sensible, remember the definition

$-\phi_{k}=\sum_{j=0}^{k} h_{i} h_{k-1}+e_{i} f_{k-1}$.

$-\phi_{0}=h_{0}^{2}+e_{0} f_{0}$, so it is not possible to replace $h_{0}$ by $\phi_{0}, e_{0}, f_{0}$. But from $h_{1}$ on, one can solve (32) for $h_{k}$ in terms of $\phi_{k}$ and the preceding $h_{j}$, which - with exception of $h_{0}$-are expressed in terms of $\phi_{j}, j<k$.

Now take the polynomial $\psi$ to be a polynomial in $e_{j}, f_{j}, j \geqslant 0 ; \phi_{j}, j \geqslant 1 ; h_{0}, h_{0}^{-1}\left(h_{0}^{-1}\right.$ enters when one solves (32) for $h_{k}$ ).

$\psi$ contains finitely many $e_{j}$ only. Let $e_{R}$ be the $e_{j}$ with highest subscript in $\psi$. So $\psi$ is of the form

$\psi=\sum_{\alpha} e_{R}^{\alpha} \rho_{a}$,

where $\rho_{\alpha}$ is a polynomial involving at most $e_{j}$ with $j \leqslant R-1$. From $\left\{\psi, \phi_{N}\right\}=0$ follows

$0=\sum_{\alpha} \alpha\left\{e_{R}, \phi_{N}\right\} e_{R}^{\alpha-1} \rho_{\alpha}+e_{R}^{\alpha}\left\{\rho_{\alpha}, \phi_{N}\right\}$

There is a term $2 h_{0} \Sigma_{\alpha} \alpha e_{R+N} e_{R}^{\alpha-1} \rho_{\alpha}$ on the right, coming from $\partial e_{R} / \partial t_{N}$ (cf. (21)). The rest of the right side involves at worst $e_{j}$ with $j \leqslant R+N-1$. So

$\sum \alpha e_{R}^{\alpha-1} \rho_{\alpha}=0$,

because there is no other way to cancel the $e_{R+N}$. Thus

$\frac{\partial}{\partial e_{R}} \sum e_{R}^{\alpha} \rho_{\alpha}=0$,

which means that $e_{R}$ in fact does not occur in $\psi$ for $R \geqslant 1$. Likewise, $f_{R}, R \geqslant 1$, is absent.

Remark. This argument does not exclude $h_{0}, e_{0}, f_{0}$, since these are in involution with $\phi_{N}$. 
It follows from this proposition that of the many variational equations for the $q(x), r(x)$ in the AKNS setup $\left(x=t_{1}\right)$, only the soliton hierarchy (9) is Hamiltonian in the $\mathscr{K}^{\perp}$ language. If we are interested in vectorfields (on $\mathscr{K}^{\perp}$ ) that commute with $\mathscr{X}_{\phi_{v}}$, therefore, we unavoidably leave our Hamiltonian framework.

Let $\mathscr{X}$ be a vectorfield commuting with $\mathscr{X}_{\phi_{N}}$ (on $\left.\mathscr{K}^{\perp}\right)$. Suppose that the components of $\partial / \partial e_{j}, \partial / \partial f_{j}$, $\partial / \partial h_{j}, j=1, \ldots, N$, are polynomial in $e, f, h$. For $j=0$, they are necessarily zero.

A new set of coordinates will be required for the description of such a vectorfield. This idea is to treat $e_{1}, \ldots, e_{N}, f_{1}, \ldots, f_{N}$ as though they are functions of $t_{N}$, and to think of all other variables as differential polynomials (w.r. to $t_{N}$ ) in these basic ones. It will be sufficient for our purposes to take the vectorfields only on the subset $\mathscr{M} \subset \mathscr{K}^{\perp}$ $\left(h_{0}=-\mathrm{i}, e_{0}=f_{0}=h_{1}=0, h^{2}+e f=-1\right.$, see 3.1$)$. On $\mathscr{M}$, one just needs the coordinates $e_{j}, f_{j}$, or the differential polynomials we will substitute for them. The relations $\phi_{k}=0$ imply inductively that the $h_{k}$ are differential polynomials of the desired form, once this has been proved for the $e$ 's and $f$ s.

Lemma. Eq. (21) may be solved to express the derivatives

$\frac{\partial^{\alpha} e_{j} \text { or } f_{j}}{\partial t_{N}^{\alpha}}, j=1, \ldots, N, \quad \alpha=0,1,2, \ldots$

as polynomials in $e_{i}, f_{i}, i \geqslant 1$; conversely the $e_{i}, f_{i}$, $i \geqslant 1$, are polynomials in these derivatives.

The proof is a straightforward induction (remember that we are working on $\mathscr{M}$ ). The vectorfield $\mathscr{X}$ on $\mathscr{M}$ is now written in the coordinates

$e_{j}^{(\alpha)}=\frac{\partial^{\alpha} e_{j}}{\partial t_{N}^{\alpha}}, \quad f_{j}^{(\alpha)}, j=1, \ldots, N, \alpha \geqslant 0:$

$\mathscr{X}=\sum_{j, \alpha} X_{j \alpha}^{e} \frac{\partial}{\partial e_{j}^{(\alpha)}}+X_{j \alpha}^{f} \frac{\partial}{\partial f_{j}^{(\alpha)}}$.

This much could be done for any $\mathscr{X}$. Since
$\left[\mathscr{X}, \partial / \partial t_{N}\right]=0$, however, the coefficients $X_{j \alpha}$ are quite special. For instance, the "integral curves" of $\mathscr{X}$ are defined by

$\left(e_{j}^{(\alpha)}\right)^{\cdot}=X_{j \alpha}^{e}$.

But

$\frac{\partial}{\partial t_{N}}\left(e_{j}^{(\alpha)}\right)=\left(\frac{\partial}{\partial t_{N}} e_{j}^{(\alpha)}\right)=\left(e_{j}^{(\alpha+1)}\right)^{\cdot}$,

so

$\frac{\partial}{\partial t_{N}} X_{j \alpha}^{e}=X_{j, \alpha+1}^{e}$

$\mathscr{X}$ is therefore determined by $2 N$ differential polynomials, which we will call $A_{j}, B_{j}(j=1, \ldots, N)$, and has the form

$\sum_{\alpha=0}^{\infty} \sum_{j=1}^{N} A_{j}^{(\alpha)} \frac{\partial}{\partial e_{j}^{(\alpha)}}+B_{j}^{(\alpha)} \frac{\partial}{\partial f_{j}^{(\alpha)}}$,

superscript $\alpha$ denoting the $\alpha$ th $t_{N}$ derivative.

In the next section, we study a formal variational calculus associated with vectorfields of the form (33).

\section{Lie algebras of vectorfields and a variational calculus}

This section is independent of the preceding ones, so we will use a neutral notation: $q_{j}^{(\alpha)}, r_{j}^{(\alpha)}$ instead of the $e_{j}^{(\alpha)}, f_{j}^{(\alpha)}$ in (33).

Let $V$ be the vectorspace generated by $q_{j}^{(\alpha)}, r_{j}^{(\alpha)}$, $j=1, \ldots, N, \alpha \geqslant 0$, let $\mathscr{B}$ be the polynomial algebra generated by these symbols, and let $\partial\left(=\partial / \partial t_{N}\right)$ be the derivation of $\mathscr{B}$ defined by

$\partial q_{j}^{(\alpha)}=q_{j}^{(\alpha+1)}, \quad \partial r_{j}^{(\alpha)}=r_{j}^{(\alpha+1)}$

As in section 2 , let $\overline{\mathscr{B}}=\mathscr{B} /$ Image $\partial$.

We want to associate Hamiltonian derivations on $\overline{\mathscr{B}}$ to certain vectorfields on $V$ in such a way that 
commuting vectorfields go to commuting derivations. This was done by Gel'fand-Dikii [15] in connection with $\mathrm{KdV}$ equation; they dealt with a differential algebra with one generator. Our generalization is conceptually straightforward, but a more awkward notation is necessary. This whole section is needed only to tie down some loose logical ends at the conclusion of the paper. The reader who skips directly to section 7 will miss little of the idea and will avoid some messy formulas.

For two vector fields* on $V$,

$$
\xi=\sum_{\alpha=0}^{\infty} \sum_{j=1}^{N}\left(a_{j}^{(\alpha)} \frac{\partial}{\partial q_{j}^{(\alpha)}}+b_{j}^{(\alpha)} \frac{\partial}{\partial r_{j}^{(\alpha)}}\right)
$$

and

$\eta=\sum_{\alpha=0}^{\infty} \sum_{j=1}^{N}\left(c_{j}^{(\alpha)} \frac{\partial}{\partial q_{j}^{(\alpha)}}+d_{j}^{(\alpha)} \frac{\partial}{\partial r_{j}^{(\alpha)}}\right)$

the commutator $[\xi, \eta]$ has $\partial / \partial q_{j}^{(\alpha)}$ component

$$
\sum_{\beta=0}^{\infty} \sum_{i=0}^{N}\left(a_{i}^{(\beta)} \frac{\partial c_{j}^{(\alpha)}}{\partial q_{i}^{(\beta)}}-c_{i}^{(\beta)} \frac{\partial a_{j}^{(\alpha)}}{\partial q_{i}^{(\beta)}}+b_{i}^{(\beta)} \frac{\partial c_{j}^{(\alpha)}}{\partial r_{i}^{(\beta)}}-d_{i}^{(\beta)} \frac{\partial a_{j}^{(\alpha)}}{\partial r_{i}^{(\beta)}}\right)
$$

and $\partial / \partial r_{j}^{(\alpha)}$ component

$$
\begin{aligned}
\sum_{\beta=0}^{\infty} & \sum_{i=0}^{N}\left(b_{i}^{(\beta)} \frac{\partial d_{j}^{(\alpha)}}{\partial r_{i}^{(\beta)}}-d_{i}^{(\beta)} \frac{\partial b_{j}^{(\alpha)}}{\partial r_{i}^{(\beta)}}+a_{i}^{(\beta)} \frac{\partial d_{j}^{(\alpha)}}{\partial q_{i}^{(\beta)}}\right. \\
& \left.-c_{i}^{(\beta)} \frac{\partial b_{j}^{(\alpha)}}{\partial q_{i}^{(\beta)}}\right) .
\end{aligned}
$$

Now we transfer vectorfields and their commutators from $V$ to $\overline{\mathscr{B}}$. Let $\alpha: \mathscr{B} \rightarrow \overline{\mathscr{B}}$ be the canonical projection, and write $\alpha(F)=\bar{F}$.

A vectorfield $\xi$ on $V$ gives rise to a derivation $\xi$ on $\overline{\mathscr{B}}$ via

$$
\bar{\xi} \bar{F}=\alpha(\xi F)
$$

This definition is correct, because $\xi$ takes $\partial \phi$ into

*From now on, all "vectorfields" are of this form: see [15] for the official language. itself. Note that (36) implies

$\overline{[\xi, \eta]}=[\bar{\xi}, \bar{\eta}]$

\section{Lemma 1.}

$$
\xi \bar{F}=\alpha\left(\sum_{j=1}^{N} a_{j}^{(0)} \frac{\delta F}{\delta q_{j}}+b_{j}^{(0)} \frac{\delta F}{\delta r_{j}}\right)
$$

Proof.

$$
\begin{aligned}
\zeta F & =\sum_{\alpha=0}^{\infty} \sum_{j=1}^{N}\left(a_{j}^{(\alpha)} \frac{\partial F}{\partial q_{j}^{(\alpha}}+b_{j}^{(\alpha)} \frac{\partial F}{\partial r_{j}^{(\alpha)}}\right) \\
& =\sum_{j=1}^{N} \sum_{\alpha=0}^{\infty} a_{j}^{(0)}(-\partial)^{\alpha} \frac{\partial F}{\partial q_{j}^{(\alpha)}}+b_{j}^{(0)}(-\partial)^{\alpha} \frac{\partial F}{\partial r_{j}^{(\alpha)}}+\partial P
\end{aligned}
$$

(for a certain $P \in \partial B$ )

$$
=\sum_{j=1}^{N}\left(a_{j}^{(0)} \frac{\delta F}{\partial q_{j}}+b_{j}^{(0)} \frac{\delta F}{\delta r_{j}}\right)+\partial P
$$

Definition.

$$
\begin{aligned}
& \Omega^{(1)}(\bar{\xi})=\alpha\left(\sum_{j=1}^{N} r_{j} a_{j}^{(0)}\right), \\
& \Omega^{(2)}(\bar{\xi}, \bar{\eta})=-\alpha\left(\sum_{j=1}^{N} b_{j}^{(0)} c_{j}^{(0)}-a_{j}^{(0)} d_{j}^{(0)}\right) .
\end{aligned}
$$

(Note that $\Omega^{(2)}$ is weakly non-degenerate on $\xi, \bar{\eta}$ ).

Lemma 2. $-\mathrm{d} \Omega^{(1)}=\Omega^{(2)}$.

Proof.

$$
\begin{gathered}
\mathrm{d} \Omega^{(1)}(\bar{\xi}, \bar{\eta})=\bar{\xi} \Omega^{(1)}(\bar{\eta})-\bar{\eta} \Omega^{(1)}(\bar{\xi})-\Omega^{(1)}([\bar{\xi}, \bar{\eta}]) \\
=\bar{\xi} \alpha\left(\sum_{j=1}^{N} r_{j} c_{j}^{(0)}\right)-\bar{\eta} \alpha\left(\sum_{j=1}^{N} r_{j} a_{j}^{(0)}\right) \\
-\alpha\left(\sum _ { j = 1 } ^ { N } r _ { j } \sum _ { k = 1 } ^ { N } \sum _ { \alpha = 0 } ^ { \infty } \left(a_{k}^{(\alpha)} \frac{\partial c_{j}^{(0)}}{\partial q_{k}^{(\alpha)}}\right.\right. \\
\left.-c_{k}^{(\alpha)} \frac{\partial a_{j}^{(0)}}{\partial q_{k}^{(\alpha)}}+b_{k}^{(\alpha)} \frac{\partial c_{j}^{(0)}}{\partial r_{k}^{(\alpha)}}-d_{k}^{(\alpha)} \frac{\partial a_{j}^{(0)}}{\partial r_{k}^{(\alpha)}}\right)
\end{gathered}
$$




$$
\begin{aligned}
= & \alpha\left(\sum_{j=1}^{N} r_{j}\left(\sum_{k=1}^{N} \sum_{\alpha=0}^{\infty} a_{k}^{(\alpha)} \frac{\partial c_{j}^{(0)}}{\partial q_{k}^{(\alpha)}}+b_{k}^{(\alpha)} \frac{\partial c_{j}^{(0)}}{\partial r_{k}^{(\alpha)}}\right)\right. \\
& \left.+\sum_{j=1}^{N} b_{j}^{(0)} c_{j}^{(0)}\right)-\alpha\left(\sum _ { j = 1 } ^ { N } r _ { j } \left(\sum_{k=1}^{N} \sum_{\alpha=0}^{\infty} c_{k}^{(\alpha)} \frac{\partial a_{j}^{(0)}}{\partial q_{k}^{(\alpha)}}\right.\right. \\
& \left.\left.+d_{k}^{(\alpha)} \frac{\partial a_{j}^{(0)}}{\partial r_{k}^{(\alpha)}}\right)+\sum_{j=1}^{k} a_{j}^{(0)} d_{j}^{(0)}\right) \\
& -\alpha(\text { last term in preceding equality }) \\
= & \alpha\left(\sum_{j=1}^{N} b_{j}^{(0)} c_{j}^{(0)}-a_{j}^{(0)} d_{j}^{(0)}\right) .
\end{aligned}
$$

Definition. For $F \in \mathscr{B}$, let

$$
\xi_{F}=\sum_{\alpha=0}^{\infty} \sum_{j=1}^{N}\left(\frac{\delta F}{\delta r_{j}}\right)^{(\alpha)} \frac{\partial}{\partial q_{j}^{(\alpha)}}-\left(\frac{\delta F}{\delta q_{j}}\right)^{(\alpha)} \frac{\partial}{\partial r_{j}^{(\alpha)}}
$$

Lemma 3. $\bar{\eta} \bar{F}=\Omega^{(2)}\left(\xi_{F}, \bar{\eta}\right)$, i.e., $\xi_{F}$ is a Hamiltonian vectorfield for the formal symplectic form $\Omega^{(2)}$.

\section{Proof.}

$\Omega^{(2)}\left(\bar{\xi}_{F}, \bar{\eta}\right)=\alpha\left(\sum_{j=1}^{N} \frac{\delta F}{\delta q_{j}} c_{j}^{(0)}+\frac{\delta F}{\delta r_{j}} d_{j}^{(0)}\right)=\bar{\eta} \bar{F}$

by lemma 1 .

Corollary. On $\overline{\mathscr{B}}$, we have a Poisson bracket

$$
\{\bar{F}, \bar{G}\}=\Omega^{(2)}\left(\xi_{F}, \xi_{G}\right)=\alpha\left(\sum_{j=1}^{N} \frac{\delta F}{\delta q_{j}} \frac{\delta G}{\delta r_{j}}-\frac{\delta F}{\delta r_{j}} \frac{\delta G}{\delta q_{j}}\right)
$$

The map $\bar{F} \mapsto \xi_{F}$ gives a Hamiltonian structure,

$$
\xi_{F}(\bar{G})=-\{\bar{F}, \bar{G}\}
$$

Proof. We compute $x_{j}^{(\alpha)}, y_{j}^{(\alpha)}$ in

$\left[\xi_{F}, \xi_{G}\right]=\sum_{\alpha=0}^{\infty} \sum_{j=1}^{N}\left(x_{j}^{(\alpha)} \frac{\partial}{\partial q_{j}^{(\alpha)}}+y_{j}^{(\alpha)} \frac{\partial}{\partial r_{j}^{(\alpha)}}\right)$

Since $\Omega^{(2)}=-\mathrm{d} \Omega^{(1)}$ is closed, $\mathrm{d} \Omega^{(2)}=0$. So

$$
\begin{aligned}
& 0=\mathrm{d} \Omega^{(2)}\left(\xi_{F}, \xi_{G}, \bar{\eta}\right)=\xi_{F} \Omega^{(2)}(\bar{\xi}, \bar{\eta})-\xi_{G} \Omega^{(2)}\left(\xi_{F}, \bar{\eta}\right) \\
& +\bar{\eta} \Omega^{(2)}\left(\bar{\xi}_{F}, \bar{\xi}_{G}\right)-\Omega^{(2)}\left(\left[\bar{\xi}_{F}, \xi_{G}\right], \bar{\eta}\right) \\
& +\Omega^{(2)}\left(\left[\xi_{F}, \bar{\eta}\right], \xi_{G}\right)-\Omega^{(2)}\left(\left[\xi_{G}, \bar{\eta}\right], \bar{\xi}_{F}\right) \\
& =-\bar{\xi}_{F} \bar{\eta} \bar{G}+\xi_{G} \bar{\eta} \bar{F}+\bar{\eta}\{\bar{F}, \bar{G}\} \\
& +\alpha\left(\sum_{j=1}^{N} y_{j}^{(0)} c_{j}^{(0)}-x_{j}^{(0)} d_{j}^{(0)}\right)+\left[\bar{\xi}_{F}, \bar{\eta}\right] \bar{G}-\left[\xi_{G}, \bar{\eta}\right] \bar{F} \\
& =-\bar{\eta} \bar{\xi}_{F} \bar{G}+\bar{\eta} \xi_{G} \bar{F}+\bar{\eta}\{\bar{F}, \bar{G}\} \\
& +\alpha\left(\sum_{j=1}^{N} y_{j}^{(0)} c_{j}^{(0)}-x_{j}^{(0)} d_{j}^{(0)}\right) \\
& =-\bar{\eta} \alpha\left(\sum _ { j = 1 } ^ { N } \left(-\frac{\delta F}{\delta r_{j}} \frac{\delta G}{\delta q_{j}}+\frac{\delta F}{\delta q_{j}} \frac{\delta G}{\delta r_{j}}+\frac{\delta G}{\delta r_{j}} \frac{\delta F}{\delta q_{j}}\right.\right. \\
& \left.\left.-\frac{\delta G}{\delta q_{j}} \frac{\delta F}{\delta r_{j}}-\frac{\delta F}{\delta q_{j}} \frac{\delta G}{\delta r_{j}}+\frac{\delta F}{\delta r_{j}} \frac{\delta G}{\delta q_{j}}\right)\right) \\
& +\alpha\left(\sum_{j=1}^{N} y_{j}^{(0)} c_{j}^{(0)}-x_{j}^{(0)} d_{j}^{(0)}\right) \\
& =-\bar{\eta}\{\bar{F}, \bar{G}\}+\alpha\left(\sum_{j=1}^{N} y_{j}^{(0)} c_{j}^{(0)}-x_{j}^{(0)} d_{j}^{(0)}\right) \\
& =(\text { by lemma } 2)-\alpha\left(\sum_{j=1}^{N} c_{j}^{(0)} \frac{\delta\{F, G\}}{\delta q_{j}}\right. \\
& \left.+d_{j}^{(0)} \frac{\delta\{F, G\}}{\delta r_{j}}-y_{j}^{(0)} c_{j}^{(0)}+x_{j}^{(0)} d_{j}^{(0)}\right) .
\end{aligned}
$$

All this started out as zero in $\overline{\mathscr{B}}$, with $c_{j}^{(0)}$ and $d_{j}^{(0)}$ arbitrary. So,

$c_{j}^{(0)}\left(\frac{\delta\{F, G\}}{\delta q_{j}}-y_{j}^{(0)}\right)$

is a perfect $\partial$-derivative for any $c_{j}^{(0)} \in \mathscr{B}$, as is $d_{j}^{(0)}\left(\delta\{F, G\} / \delta r_{j}+x_{j}^{(0)}\right)$. It follows that

$x_{j}^{(0)}=-\frac{\delta\{F, G\}}{\delta r_{j}}, \quad y_{j}^{(0)}=\frac{\delta\{F, G\}}{\delta g_{j}}$

Proposition 9. $\left[\xi_{F}, \xi_{G}\right]=0$ iff $\{\bar{F}, \bar{G}\}=0$.

Proof. By lemma 3 and eq. (39) 


$$
\begin{aligned}
{\left[\xi_{F}, \xi_{G}\right] } & =0 \quad \text { iff } \frac{\delta\{F, G\}}{\delta q_{j}}=\frac{\delta\{F, G\}}{\delta r_{j}}=0, \\
j & =1, \ldots, N
\end{aligned}
$$

so that

$\left[\xi_{F}, \xi_{G}\right]=0 \quad$ iff $\{F, G\} \in \operatorname{Image} \partial \operatorname{iff}\{\bar{F}, \bar{G}\}=0$.

\section{Lax equations as Hamiltonian derivations}

The theory of the last section is now applied to certain vectorfields on $\mathscr{K}^{\perp}$ that commute with $\mathscr{X}_{\phi_{N}}$ : namely, vectorfields of the form $\xi_{F}$, with $F$ a differential polynomial in $e_{1}, \ldots, e_{N}, f_{1}, \ldots, f_{N}$ (playing the role of $q_{j}, r_{j}$ ). In particular, all $X_{\phi_{j}}$ will be shown to be of this form, so that (by prop. 9) they map to commuting Hamiltonian derivations on $\overline{\mathscr{B}}$ (the derivation $\partial$ is $\partial / \partial t_{N}$, as explained already).

It turns out that the most convenient variables $q_{j}, r_{j}$ are in fact not the $e_{j}, f_{j}$ themselves, but rather certain polynomials in the $e_{j}, f_{j}$. Define formal series

$\tilde{e}=\frac{e}{\sqrt{i-h}}=\sum_{j=1}^{\infty} \tilde{e}_{j} \zeta^{-j}, \quad \tilde{f}=\frac{f}{\sqrt{i-h}}=\sum_{j=1}^{\infty} \tilde{f}_{j} \zeta^{-j}$

subject to $h^{2}+e f=-1$ and $h_{0}=-\mathrm{i}$, $e_{0}=f_{0}=h_{1}=0$. It is clear that $\tilde{e}_{k}$ and $\tilde{f}_{k}$ are polynomials in $e_{j}, f_{j}, j \leqslant k$, and conversely $e_{k}$ and $f_{k}$ are polymomials in $\tilde{e}_{j}, f_{j}, j \leqslant k$. Specifically,

$\tilde{e}_{k}=\frac{1}{\sqrt{2 \mathrm{i}}} e_{k}+$ polynomial in $e_{j}, f_{j}, j \leqslant k-1$,

and likewise for $\tilde{f}_{k}$. We may therefore use the $\tilde{e}_{k}, \tilde{f}_{k}$ as coordinates on $\mathscr{H}$, and along the lines of section 6, we may write vectorfields commuting with $\mathscr{X}_{\phi_{N}}$ in the form (33) with twiddles over $e_{j}^{(\alpha)}$ and $f_{j}^{(\alpha)}$.

$\dagger$ We discovered $\tilde{e}$ and $\tilde{f}$ in the course of work on the stationary AKNS equations in 1980, more or less by accident, after long calculations with formal series. There is still no satisfactory explanation of their origin, nor a good theoretical reason for their significance. They appear again in paper III. $\tilde{e}$ and $\tilde{f}$ are not particularly convenient for the study of the Hamiltonian structure on $\mathscr{K}^{\perp}$, but they are fundamental in the variational Hamiltonian framework, as we shall see in this sectiont.

Our object is to calculate the variational derivatives of the $F_{k j}$ with respect to $\tilde{e}_{j}, \tilde{f}_{j}, j=1, \ldots, N$, where the $F_{k j}$ are considered to be differential polynomials in $t_{N}$-derivatives of these $\tilde{e}_{j}, \tilde{f}_{j}$. This will be done in one fell swoop, via generating series. The calculation is based on a characterization of functional derivatives used to great advantage by Gel'fand-Dikii in the study of stationary $\mathrm{KdV}$ equations [16].

Let $F \in \mathscr{B}$, the (polynomial) differential algebra with generators $q_{j}, r_{j}, j=1, \ldots, N$ and derivation $\mathrm{d} / \mathrm{d} x$. There exists a unique 1 -form $\omega^{(1)}$, and unique $A_{j}, B_{j} \in B$, such that

$\mathrm{d} F=\sum_{j=1}^{N} A_{j} \mathrm{~d} q_{j}+B_{j} \mathrm{~d} r_{j}+\frac{\mathrm{d}}{\mathrm{d} x} \omega^{(1)}$.

(The exterior derivative $d$ has the natural properties, and it commutes with $d / d x$; see [17] for definitions and a proof of (41)). Furthermore,

$A_{j}=\frac{\delta F}{\delta q_{j}}, \quad B_{j}=\frac{\delta F}{\delta r_{j}}$

according to our earlier definition in section 2 .

The idea behind (41) is of course the familiar calculation in the calculus of variations,

$$
\begin{aligned}
\delta \int F \mathrm{~d} x= & \int\left(\sum \frac{\delta F}{\delta q_{j}} \delta q_{j}+\frac{\delta F}{\delta r_{j}} \delta r_{j}\right. \\
& + \text { perfect } x \text {-derivative }) \mathrm{d} x
\end{aligned}
$$

We will prove the following formula:

\section{Theorem 2.}

$$
\begin{aligned}
\mathrm{d} F_{N k}= & -\frac{\mathrm{i}}{2} k \sum_{j+l=N+1} \frac{\partial \tilde{f}_{j}}{\partial t_{k-1}} \mathrm{~d} \tilde{e}_{l}-\frac{\partial \tilde{e}_{j}}{\partial t_{k}} \mathrm{~d} \tilde{f}_{l} \\
& +\frac{\partial}{\partial t_{N}}\left(-\frac{\mathrm{i}}{2}\left(\tilde{f}_{\zeta} \mathrm{d} \tilde{e}-\tilde{e}_{\zeta} \mathrm{d} \tilde{f}\right)\right)_{k+1}
\end{aligned}
$$


Explanation. $F_{N k}, k \geqslant 1$, is thought of as differential $t_{N}$-polynomial in $\tilde{\boldsymbol{e}}_{j}, \tilde{J}_{j}, j=1, \ldots, N$. Subscript ()$_{k+1}$ denotes the coefficient of $\zeta^{-k-1}$ in a formal series, and subscript $\zeta$ denotes $\mathrm{d} / \mathrm{d} \zeta$. $\partial \tilde{e}_{j} / \partial t_{k-1}$ denotes the differential $t_{N}$-polynomial obtained when this derivative is calculated according to (21) and (40). Always, $h_{0}=-\mathrm{i}, h_{1}=e_{0}=f_{0}=0$, $h^{2}+e f=-1$.

From (42) and (41), we get

$\frac{\partial \tilde{f}_{j}}{\partial t_{k-1}}=\frac{2 \mathrm{i}}{k} \frac{\delta F_{N k}}{\delta \tilde{e}_{N+1-j}}$

$\frac{\partial \tilde{e}_{j}}{\partial t_{k-1}}=-\frac{2 \mathrm{i}}{k} \frac{\delta F_{N k}}{\delta \tilde{f}_{N+1-j}}$

So we have - informally - the third main result advertised in the introduction: the $t_{k-1}$-flow $(k \geqslant 1)$ is Hamiltonian in the differential algebra of "functions of $t_{N}$ ". The Hamiltonian is $(2 \mathrm{i} / k) F_{N k}$, and the conjugate variables are $\tilde{f}_{j}$ and $\tilde{e}_{N+1-j}$, $j=1, \ldots, N$. Some details are needed to connect the various algebraic structures in a rigorous way.

We will first prove theorem 2 ; this requires a lengthy and - for a change - somewhat subtle calculation. Then we bring in the variational Hamiltonian framework of the preceding section to explain more fully the transition from $\mathscr{K}^{\perp}$ to a differential algebra.

Proof of theorem $2 . \dagger$ We introduce the differentials $\mathrm{d} e_{j}, \mathrm{~d} f_{j}$ and their generating series $\mathrm{d} e, \mathrm{~d} f$. It will be necessary to calcuate expressions of the form

$\left\{h^{2}+e f, \mathrm{~d} e^{\prime}\right\}$

$\left(e^{\prime}=e(\eta)\right)$. By general principles, $\mathrm{d}$ commutes with any Lie derivative $\mathscr{L}_{\mathscr{X}}$, so

† The proof has nothing to do with Lie algebras; we realize that an intrinsic argument is desirable, and hope to be able to provide one eventually. This is the only important result of the paper we do not yet have for arbitrary simple $g$ in place of $s l(2)$.

$$
\begin{aligned}
\left\{h^{2}+e f, \mathrm{~d} e^{\prime}\right\} & =\sum \mathscr{L}_{x_{\phi_{k}}} \mathrm{~d} e_{j} \zeta^{-k} \eta^{-j} \\
& =\sum \mathrm{d} \frac{\partial e_{j}}{\partial t_{k}} \zeta^{-k} \eta^{-j}=\mathrm{d}\left\{h^{2}+e f, e^{\prime}\right\}
\end{aligned}
$$

The first relation to be derived is

$$
\begin{gathered}
-\frac{2 \eta^{-2} \zeta^{-1}}{\left(\zeta^{-1}-\eta^{-1}\right)^{2}} \mathrm{~d}\left(e f^{\prime}+e^{\prime} f+2 h h^{\prime}+2\right) \\
=\left\{h^{2}+e f, f^{\prime}\right\}_{\eta} \frac{\mathrm{d} e}{h}-\left\{h^{2}+e f, e^{\prime}\right\}_{\eta} \frac{\mathrm{d} f}{h} \\
+\left\{h^{2}+e f, \frac{e_{\eta}^{\prime} \mathrm{d} f^{\prime}-f_{\eta}^{\prime} \mathrm{d} e^{\prime}}{h^{\prime}}\right\} .
\end{gathered}
$$

To check this, calculate $\left\{h^{2}+e f\right.$, $\left.\left(e_{\eta}^{\prime} \mathrm{d} f^{\prime}-f_{\eta}^{\prime} \mathrm{d} e^{\prime}\right) / h^{\prime}\right\}$ using (22) and (44). (Subscript $\eta$ denotes $\mathrm{d} / \mathrm{d} \eta$ ). Calculate the other terms from (22), and simplify, using the relations

$2 h \mathrm{~d} h+e \mathrm{~d} f+f \mathrm{~d} e=0, \quad 2 h^{\prime} h_{\eta}^{\prime}+e^{\prime} f_{\eta}^{\prime}+f^{\prime} e_{\eta}^{\prime}=0$

that follow from $h^{2}+e f=-1$.

Remark. The tricky part of (45) is guessing the 1-form $\left(h^{\prime}\right)^{-1}\left(e_{\eta}^{\prime} \mathrm{d} f^{\prime}-f_{\eta}^{\prime} \mathrm{d} e^{\prime}\right)$. It is the counterpart of the 1-form used by Gel'fand-Dikii in [16]; compare their equation (4.4).

The left side of (45) is $4 \mathrm{i} \eta^{-1}$ times $\mathrm{d}$ of the generating function (27) of the $F_{k j}$, which is what is wanted on the left side of (42).

We must now replace $e^{\prime}, f^{\prime}$ on the right by $\tilde{e}^{\prime}, \tilde{f}^{\prime}$.

To this end, we observe that $\eta\left\{h^{2}+\right.$ $\left.e f, e^{\prime}\right\}=2\left(e h^{\prime}-e^{\prime} h\right) /\left(\zeta^{-1}-\eta^{-1}\right)$ is symmetric in $\zeta$, $\eta$, so that

$\eta\left\{h^{2}+e f, e^{\prime}\right\}=\zeta\left\{h^{\prime 2}+e^{\prime} f^{\prime}, e\right\}$,

with a similar expression for $\left\{, f^{\prime}\right\}$.

The right side of (45) becomes, after $\left\{h^{2}+e f, e^{\prime}\right\} \mapsto \eta^{-1} \zeta\left\{h^{\prime 2}+e^{\prime} f^{\prime}, e\right\}$,

$$
\begin{aligned}
& {\left[\eta^{-1} \zeta\left(\left\{h^{\prime 2}+e^{\prime} f^{\prime}, f\right\} \frac{\mathrm{d} e}{h}-\left\{h^{\prime 2}+e^{\prime} f^{\prime}, e\right\} \frac{\mathrm{d} f}{h}\right)\right]_{\eta}} \\
& +\left\{h^{2}+e f, \frac{e_{\eta}^{\prime} \mathrm{d} f^{\prime}-f_{\eta}^{\prime} \mathrm{d} e^{\prime}}{h^{\prime}}\right\} .
\end{aligned}
$$


Now replace $e$ by $\tilde{e}(\mathrm{i}-h)^{1 / 2}$ :

$$
\begin{aligned}
\begin{aligned}
\left\{h^{\prime 2}+e^{\prime} f^{\prime}, e\right\}= & (\mathrm{i}-h)^{1 / 2}\left\{h^{\prime 2}+e^{\prime} f^{\prime}, \tilde{e}\right\} \\
& -\frac{1}{2}(\mathrm{i}-h)^{-1 / 2} \tilde{e}\left\{h^{\prime 2}+e^{\prime} f^{\prime}, h\right\},
\end{aligned} \\
\mathrm{d} e=(\mathrm{i}-h)^{1 / 2} \mathrm{~d} \tilde{e}-\frac{1}{2}(\mathrm{i}-h)^{-1 / 2} \tilde{e} \mathrm{~d} h .
\end{aligned}
$$

From $h^{2}+e f=-1$ one finds

$h+\mathrm{i}=\frac{e f}{\mathrm{i}-h}=\tilde{e} \tilde{f}$,

whence

$\mathrm{d} h=\tilde{e} \mathrm{~d} \tilde{f}+\tilde{f} \mathrm{~d} \tilde{e}$,

which is plugged into (48).

Another consequence of $h^{2}+e f=-1$ is

$\left\{h^{\prime 2}+e^{\prime} f^{\prime}, h\right\}=\tilde{e}\left\{h^{\prime 2}+e^{\prime} f^{\prime}, \tilde{f}\right\}+\tilde{f}\left\{h^{\prime 2}+e^{\prime} f^{\prime}, \tilde{e}\right\}$

which is plugged into (47). Similar things are done to $f=f(\mathrm{i}-h)^{1 / 2}$.

A straightforward calculation now turns (46) into

$$
\begin{aligned}
& {\left[-2 \eta^{-1} \zeta\left(\left\{h^{\prime 2}+e^{\prime} f^{\prime}, \tilde{f}\right\} \mathrm{d} \tilde{e}-\left\{h^{\prime 2}+e^{\prime} f^{\prime}, \tilde{e}\right\} \mathrm{d} \tilde{f}\right)\right]_{\eta}} \\
& \quad+2\left\{h^{2}+e f, \tilde{f}_{\eta}^{\prime} \mathrm{d} \tilde{e}^{\prime}-\tilde{e}_{\eta}^{\prime} \mathrm{d} \tilde{f}^{\prime \prime}\right\}
\end{aligned}
$$

This is substituted for the right side of (45). Next, multiply (45) by $-i / 4$; the left side is then

$\sum_{k=1, j=1}^{\infty} \mathrm{d} F_{k j} \eta^{-k-1} \zeta^{-j}$

On the new right side, substitute the series

$\left\{h^{\prime 2}+e^{\prime} f^{\prime}, \tilde{e}\right\}=\sum_{k=0, j=1}^{\infty} \frac{\partial \tilde{e}_{j}}{\partial t_{k}} \eta^{-k \zeta^{-j}}$, etc.

and match powers. The desired formula (42) drops out.
There is a point of logic to be disposed of before theorem 2 can be applied. In (42), $d$ is the exterior derivative with respect to the variables $e_{j}, f_{j}, j \geqslant 1$, on $\mathscr{M}$. In (41), with which (42) was compared to give the Hamiltonian equations (43), $\mathrm{d}$ is an exterior derivative defined on the generators $q_{j}, r_{j}$ and extended to all of $\mathscr{B}$ so as to commute with the distinguished derivation $\mathrm{d} / \mathrm{d} x$. It had better be the case, therefore, that the differentials $\mathrm{d} F_{N k}$ and $\left(\partial / \partial t_{N}\right)\left(\tilde{f}_{\zeta} \mathrm{d} \tilde{e}-\tilde{e}_{\zeta} \mathrm{d} \tilde{f}\right)_{k+1}$ in (42) can be calculated in two ways:

Express them in terms of $\mathrm{d} \tilde{e}_{j}, \mathrm{~d} \tilde{f}_{j}, j \geqslant 1$, then replace the $\tilde{e}_{j}, \tilde{f}_{j}$ by appropriate differential polynomials and pass to the differential-algebra basis $\mathrm{d} \tilde{e}_{j}^{(\alpha)}, \mathrm{d} \tilde{f}_{j}^{(\alpha)}, j=1, \ldots, N$, or

Express everything as polynomials in $\tilde{e}_{j}^{(\alpha)}, \tilde{f}_{j}^{(\alpha)}$, $j=1, \ldots, N$, and then apply $\mathrm{d}$, requiring it to commute with $\partial / \partial t_{N}$.

The reader may convince himself that these procedures are indeed equivalent; the key is the commutativity of $\mathrm{d}$ and $\mathscr{L}_{\boldsymbol{x}_{\phi_{\mathrm{N}}} \text {. }}$

Now we can describe quite precisely what happens to our $t_{k}$-flows when $t_{N}$ is singled out.

$\mathscr{X}_{\phi_{k}}$ is a vectorfield that commutes with $\mathscr{X}_{\phi_{N}}$, and so it has the form (33),

$X_{\phi_{k}}=\sum_{\alpha=0}^{\infty} \sum_{j=1}^{N} A_{j}^{(\alpha)} \frac{\partial}{\partial \tilde{e}_{j}^{(\alpha)}}+B_{j}^{(\alpha)} \frac{\partial}{\partial \tilde{f}_{j}^{(\alpha)}}$

The coefficients $A_{j}^{(0)}, B_{j}^{(0)}$ are of course exactly the differential polynomials we have denoted by

$\frac{\partial \tilde{e}_{j}}{\partial t_{k}}, \frac{\partial \tilde{f}_{j}}{\partial t_{k}}$

By (43), these are functional derivatives

$\frac{\partial \tilde{e}_{j}}{\partial t_{k}}=-\frac{2 \mathrm{i}}{k+1} \frac{\delta F_{N, k+1}}{\delta \tilde{f}_{N+1-j}}$,

$\frac{\partial \tilde{f}_{j}}{\partial t_{k}}=\frac{2 \mathrm{i}}{k+1} \frac{\delta F_{N, k+1}}{\delta \tilde{e}_{N+1-j}}$.

Hence the vectorfield $\mathscr{X}_{\phi_{k}}$ on $\mathscr{K}^{\perp}$ gives rise to a vectorfield $\xi_{F_{k}}$ on the differential algebra (cf 
section 6), with $F_{k}=2 \mathrm{i} /(k+1) F_{N, k+1}$. This induces a Hamiltonian derivation $\xi_{F_{k}}$ on $\overline{\mathscr{B}}$. Finally, since the $\mathscr{X}_{\phi_{k}}$ commute with each other on $\mathscr{K}^{\perp}$, the corresponding $\xi_{F_{k}}$ commute on $\mathscr{B}$, and by proposition 9, the variational Hamiltonians $\bar{F}_{k}$ are in involution with respect to the natural Poisson bracket on $\overline{\mathscr{B}}$.

\section{Summary}

We conclude this paper with a restatement of the overall logical thread of our approach.

1) The starting point is an infinite-dimensional Lie algebra. We think of this as having been obtained from a given equation by use of the ideas of Wahlquist-Estabrook. It is generally thought that the existence of a nontrivial infinite prolongation algebra indicates that the equation belongs to an infinite family of commuting flows.

2) The Lie algebra is written as a direct sum, an inner product is introduced and certain adinvariant functions are identified; this is the Adler-Kostant-Symes setup. These functions are the Hamiltonians for our infinite set of commuting flows, $Q_{t_{k}}=\left[Q^{(k)}, Q\right], k=0,1, \ldots$.

3) The Lax form of these equations immediately suggests that $Q$ may be written $Q=V Q_{0} V^{-1}$, where $V_{t_{k}}=Q^{(k)} V$, a form which reveals the group theoretic nature of $V$. Thus, the auxiliary functions $V$ (the old eigenfunctions) are introduced after the commuting flows. If the Lie algebra is graded, as it is in this paper, $\zeta$, which is introduced as the grading parameter, becomes the "eigenvalue". However, up to this point no particular $t_{k}$ is distinguished. If we distinguish $t_{1}=x$ and take $V_{t_{1}}=Q^{(1)} V$ to be the eigenvalue problem, then we recover the AKNS flows. If we were to distinguish $t_{2}$ and pick $V_{t_{2}}=Q^{(2)} V$ to be the eigenvalue problem, there are four dependent variables $\left(e_{1}, e_{2}, f_{1}, f_{2}\right)$ and a different set of flows is found, in a new function space. As one example, we take the special case $e_{2}=f_{2}=0, f_{1}=-\bar{e}_{1}$, with everything independent of $t_{2 N+1}, N=0,1, \ldots$ Then the $t_{4}$ flow is the derivative nonlinear Schrödinger equation [19]

$q_{t_{4}}=\mathrm{i} q_{t_{2} l_{2}}+\left(q^{2} \bar{q}\right)_{t_{2}}$

when

$e_{1}=q \mathrm{e}^{-\mathrm{i} \int_{-\infty}^{\infty} q \bar{q} \mathrm{~d} t_{2}}$

4) The properties 1), 3), 5) and (we conjecture) 7) follow without resort to the auxiliary $V$ equations. Properties 1), 3) have already been discussed in this paper, and the Hirota $\tau$-function was introduced (sketchily). In paper IV, we discuss how the equations (21) for the $h_{j}, e_{j}, f_{j}$, with each variable thought of as $\partial / \partial t_{j}$-derivative of a potential (see (23)), can be written equivalently as equations for the potentials $\tau, \sigma=e_{1} \tau, \rho=f_{1} \tau$. These are the Hirota equations.

5) On the other hand, results about Bäcklund transformations follow more naturally from the auxiliary equations for the $V$ 's. One knows, for example, what property $V$ must have if it is to be derived from a $Q$ which contains a soliton with given parameters. Multiplying $V$ on the left by a matrix $R$ to add a soliton is equivalent to multiplying $Q$ on the left by $R$ and on the right by $R^{-1}$. The new $\tilde{Q}=R Q R^{-1}$ obeys the same equations in $t_{1}, t_{2}, \ldots$ as the old $Q$. Thus the equation $R Q=\tilde{Q} R$ provides the Bäcklund transformation between solutions of all equations in the family. We develop this point in more detail, and work out examples, in paper IV.

It is not yet clear to us whether this material generalizes beyond replacement of $\operatorname{sl}(2)$ by $g$. Since our considerations are entirely local in the independent variables, there is in principle no exclusion of "higher space dimension" problems. The work of the Kyoto group on the Kadomtsev-Petviashvilii hierarchy indicates very clearly that the appropriate phase space is a subalgebra of $\mathrm{gl}(\infty)$. We hope that eventually we will be able to identify from an algorithmic standpoint the appropriate algebra, its splitting, and the Hamiltonian framework, for all the soliton equations. 


\section{Acknowledgements}

This work is an outgrowth of our efforts to relate the new theory of $\tau$-functions and vertex operators to earlier soliton mathematics; the problems might never have been posed had not Flaschka been at the Kyoto University RIMS when the results reported in [5] were being discovered. Flaschka and (through him) the other authors are indebted to $\mathrm{E}$. Date, M. Jimbo, M. Kashiwara, T. Miwa, and M. and $\mathrm{Y}$. Sato for many explanations. We also wish to acknowledge the support of NSF and DOA, which made our collaboration possible.

\section{References}

[1] V.G. Drinfel'd and V.V. Sokolov, Dokl. Akad. Nauk SSSR 258:1 (1981) 11 .

[2] G. Wilson, Ergodic Theory and Dynam. System 1 (1981) 361.

[3] B. A. Kupershmidt and G. Wilson, Invent. Math. 62 (1981) 403.
[4] R. Hirota, Phys. Rev. Letters 27 (1971) 1192.

[5] M. Kashiwara and T. Miwa, Proc. Japan Acad. 57A (1981) 342; E. Date, M. Kashiwara and T. Miwa, ibid. 57A (1981) 387; E. Date, M. Jimbo, M. Kashiwara and T. Miwa, J. Phys. Soc. Japan 50 (1981) 3806, 3813; Physica 4D (1982) 343; RIMS preprints 360 (1981) 362.

[6] G. Segal and G. Wilson, Loop groups and equations of KdV type, 1983 preprint.

[7] J. Palmer and D. Pickerell, in preparation.

[8] P. Deift and E. Trubowitz, Comm. Pure Appl. Math. 32 (1979) 121.

[9] P.F. Dhooghe, Bäcklund equations on Kac-Moody-Lie algebras and integrable systems, 1982 preprint.

[10] M. Adler and P. van Moerbeke, Adv. Math. 38 (1980) 267.

[11] M. Adler, Invent. Math. 50 (1979) 219.

[12] B. Kostant, Adv. Math. 34 (1979) 195.

[13] W.W. Symes, Invent. Math 59 (1980) 13.

[14] T. Ratiu, in: Springer Lecture Notes in Mathematics V. (1980) 219.

[15] I.M. Gel'fand and L.A. Dikii, Funkts. Anal. Prilozh. 10:1 (1976) 26.

[16] I.M. Gel'fand and L.A. Dikii, Funkts. Anal. Prilozh. 13:1 (1979) 8.

[17] I.M. Gel'fand and L.A. Dikii, Uspekhi Mat. Nauk 30:5 (1975) 67.

[18] I.M. Gel'fand, Yu.I. Manin and M.A. Shubin, Funkts. Anal. Prilozh. 10:4 (1976) 30.

[19] D. J. Kaup and A.C. Newell, J. Math. Phys. 19 (1978) 798. 